\title{
Preliminaries toward studying resonant extraction from the Debuncher
}

\author{
Leo Michelotti and John Johnstone \\ June 1, 2009 ( rev. March 12, 2010)
}

The real trouble with this world of ours is not that it is an unreasonable world, nor even that it is a reasonable one. The commonest kind of trouble is that it is nearly reasonable, but not quite. ... It looks just a little more mathematical and regular than it is ....

— Gilbert Keith Chesterton

Orthodoxy

\section{Introduction}

A recent proposal to detect $\mu \rightarrow e$ direct conversion at Fermilab asks for slow extraction of protons from the antiproton source, specifically from the Debuncher. [1] A third-integer resonance originally was considered for this, partly because of the Debuncher's three-fold symmetry and partly because its operational horizontal tune, $v_{x} \approx 9.765$, is already within 0.1 of $v_{x}=29 / 3$. Using a half integer resonance, $v_{x}=19 / 2$, though not part of the original proposal, has been suggested more recently because (a) Fermilab has had a good deal of experience with half-integer extraction from the Tevatron, the Main Injector and the erstwhile Main Ring, and (b) for reasons we shall examine later, it depopulates the entire bunch without an abort at the end.

This memo presents considerations preliminary to studying both possibilities. It is meant only as a starting point for investigations to be carried out in the future. The working constraints and assumptions have oscillated between two extremes: (1) making minimal changes in the antiproton source to minimize cost and (2) building another machine in the same tunnel. In this memo we adopt an attitude aligned more toward the first. The assumed parameters are listed in Table 1. A few are not (easily) subject to change, such as those related to the beam's momentum and revolution frequency and the acceptance of the debuncher.

Two resonance exemplars ${ }^{1}$ are presented in the next section, with an explanation of the analytic and semi-analytic calculations that can be done for each. Section 3 contains preliminary numerical work that was done to validate the exemplars within the context of extraction from the Debuncher. A final section contains a summary. Following the bibliography, appendices contain (a) a qualitative, conceptual discussion of extraction for the novice, (b) a telegraphic review of the perturbative incantations used to filter the exemplars as principal resonances of quadrupole, sextupole and

\footnotetext{
${ }^{1}$ While it sounds awkward, "exemplar" (or "exemplary cause" or "exemplary form") is the correct word in this context. According to Kreyche [2, pp.260-261], “[T] act intelligently means to measure or gauge one's activity according to the knowledge one has of a form. ... St.Thomas [Aquinas (De Veritate, Q.3 a.1)] defines 'exemplary cause' thus: 'it is a "form in imitation of which something comes into being from the intention of an agent that determines its end for itself.", " That pretty much says it all.
} 


\begin{tabular}{llll} 
Quantity & Unit & Model & Actual $^{*}$ \\
\hline Kinetic energy & $\mathrm{Gev}$ & 8 & \\
Momentum & $\mathrm{GeV} / \mathrm{c}$ & 8.88889 & 8.88626 \\
$B \rho$ & $\mathrm{T}-\mathrm{m}$ & 29.6501 & \\
$(\beta, \gamma, \beta \gamma)$ & & $(0.994,9.526,9.474)$ & \\
Circumference & $\mathrm{m}$ & 505.283 & 505.294 \\
Rev. period & $\mu \mathrm{sec}$ & 1.695 & \\
Rev. frequency & $\mathrm{kHz}$ & 590.038 & 590.018 \\
$\mathrm{~N}_{\text {turns }} / 60 \mathrm{~Hz}$ & & 9834 & \\
Acceptance & $\pi \mathrm{mm}-\mathrm{mr}$ & & \\
Initial emittance & $\pi \mathrm{mm}-\mathrm{mr}$ & $20-25 / \beta \gamma$ \\
Septum gap & $\mathrm{cm}$ & 1.6 & \\
Wire width & $\mu \mathrm{m}$ & 100 & \\
$\left(\beta_{x}, \beta_{y}\right)$ at septum & $\mathrm{m}$ & $(14.5,5.2)$ & \\
$\left(\beta_{x}, \beta_{y}\right)$ at lambertson & $\mathrm{m}$ & $(8.2,10.4)$ & \\
$\left(\beta_{x}, \beta_{y}\right)$ at sextupoles & $\mathrm{m}$ & $\approx(14,4-6)$ & \\
Resonant tunes & & $29 / 3$ (third integer) & \\
& & $19 / 2$ (half integer) & \\
& & &
\end{tabular}

* These four numbers were taken from Steve Werkema's slides, presented November 18.

Table 1: Parameters for slow extraction from Debuncher.

octupole distributions, (c) a brief discussion of linearly independent control circuits, and (d) two files describing the antiproton source's rings in MAD v.8 format, not readily available elsewhere. ${ }^{2}$ All figures are located at the end.

We emphasize again, the work reported here barely begins the effort that will be required to design, validate and perform resonant extraction from the Debuncher. Our goal was to compile these preliminary notes in one place for easy future reference, preferably by a young, intelligent, motivated and energetic graduate student.

\section{Analysis of two resonance exemplars}

Resonance exemplars are fictitious, integrable, autonomous Hamiltonian dynamical systems that can be connected with more realistic models using incantations derived from classical perturbation theory. A minimal exposition of these incantations has been relegated to Appendix B, to which you are referred for an explanation of the notation used in this memo. The examplars are useful and reasonably valid only "near resonance" and only in certain physical situations, to be made more precise later.

In this section we shall examine the forms given in Equations (1) and (14) and connect their control parameters with properties that can be expressed analytically: (a) the resonant orbit, (b) its separatrix, (c) emittance of the central stable region, (d) step size, and (e) spill rate. To the best of our knowledge, these calculations were first done by Teng [3] and Edwards [4] and have since been repeated and extended in various contexts, e.g. by one [5] or the other [6] of the current authors for half-integer extraction. ${ }^{3}$ The motivations for rewriting them here and in this form are:

(a) one of us (LM) needed this review,

\footnotetext{
${ }^{2}$ Steve Werkema had begun writing similar files some years ago.

${ }^{3}$ Ohnuma's 1972 memo [7] contained results from simulations but no analytical expressions.
} 
(b) to write everything explicitly using polynomials in the linear normal form coordinates appropriate for later use at higher orders in perturbation theory, and

(c) to compile in one place a minimal set of expressions that might be useful to "jumpstart" a fresh graduate student intent on seriously studying the extraction process.

There are two different, but parallel approaches for optical studies: one, discrete (maps), the other, continuous (flows). ${ }^{4}$ We shall use flows, because (i) we assume, based on experience, that most readers ${ }^{5}$ are more comfortable with this approach, and (ii) the analytic expressions we need are most easily obtained using Hamiltonian exemplars. Accordingly, the discussion will be carried out in the language of Hamilton's differential equations. Further, our meditations are confined to one degree of freedom. Even when the subscript $x$ does not appear explictly, it is assumed that all symbols are referred to the horizontal plane.

\subsection{Third integer}

The principal exemplar of the third integer resonance is written in Eq.(41) of Appendix B and transcribed below.

$$
H=\Delta \cdot a^{*} a-i g a^{3}+i g^{*} a^{* 3}+\cdots
$$

With one exception ${ }^{6}$, the notation is that of Appendix B (esp. see Eq.(29)); $\Delta=v_{x}-29 / 3 \approx 0$ is the difference between the linear (small amplitude) horizontal tune and the resonant tune and is presumed to be nearly zero; and the "resonance coupling constant," $g$, is a linear functional of the sextupole field strength distribution, as specified in Eq.(38).

$$
g=i G_{3}=\frac{i}{6 \sqrt{2}} \frac{1}{4 \pi} \sum e^{i n \theta}\left(\frac{B^{\prime \prime} l}{B \rho} \beta_{1}^{3 / 2}(\theta) e^{-i 3\left(\psi_{1}(\theta)-v_{1} \theta\right)}\right)
$$

Two basic assertions are that (a) everything, expressed by the "..." symbol, can be transformed away using standard techniques from perturbation theory ${ }^{7}$ and (b) as $\Delta \rightarrow 0$, the transformation becomes the identity and therefore is (more or less) ignorable.

Resonant orbit. Those vertices are the resonant orbit, or, in the "co-moving frame," the fixed points of Hamilton's equation of motion.

$$
\begin{aligned}
& d a / d \theta=-i \partial H / \partial a^{*}=-i \Delta a+3 g^{*} a^{* 2}+\cdots \\
& d a / d \theta=0 \quad \Longrightarrow \quad \Delta=-3 i g^{*} a_{0}^{* 2} / a_{0}
\end{aligned}
$$

Using Eq.(29) and defining the phase, $\Psi$,

$$
g \equiv|g| e^{i \Psi}
$$

gives us,

$$
\Delta=3|g|\left|a_{0}\right| e^{i\left(3 \varphi_{0}-\Psi\right)}
$$

\footnotetext{
${ }^{4}$ They are connected via exponentiation; the Hamiltonian (at one point in the ring) is pulled from the map via a process analogous to taking a logarithm. For details, read the superb textbooks of Michelotti [8] and Forest [9].

${ }^{5}$ Making a highly optimistic assumption of their existence.

${ }^{6}$ One piece of notation in this section is different from that of Section B, which is where Eq.(38) appears. I apologize for that oversight but do not intend to fix it. Effectively, $i G_{3}=g$.

${ }^{7}$ Those techniques were explained ad nauseam in both "flow" and "map" contexts in Chapters 4 and 5 of Intermediate Classical Dynamics [8]. We shall not repeat that material here.
} 
Because $\Delta$ must be real, we get,

$$
\begin{aligned}
\left|a_{0}\right|=\sqrt{I_{0}} & =|\Delta / 3 g| \\
0<\Delta \Rightarrow \varphi_{0} & =\Psi / 3 \bmod 2 \pi / 3 \\
\Delta<0 \Rightarrow \varphi_{0} & =\Psi / 3+\pi / 3 \quad \bmod 2 \pi / 3 .
\end{aligned}
$$

Separatrix geometry. That the separatrix consists of three straight lines intersecting at the vertices of an equilateral triangle is undisputed, but we shall verify it notwithstanding. The value of the Hamiltonian on the separatrix is the value it assumes on the unstable resonant orbit. So, using Equations (1), (3) and (5), we evaluate on the separatrix,

$$
\begin{aligned}
H\left(a, a^{*}\right)=H\left(a_{0}, a_{0}^{*}\right) \equiv H_{0} & =\frac{1}{3}\left(\Delta \cdot a_{0}^{*}-3 i g a_{0}^{2}\right) a_{0}+\frac{1}{3}\left(\Delta \cdot a_{0}+3 i g a_{0}^{* 2}\right) a_{0}^{*}+\frac{1}{3}\left(\Delta \cdot a_{0}^{*} a_{0}\right) \\
& =\frac{1}{3} \Delta \cdot\left|a_{0}\right|^{2} \\
& =\frac{1}{27} \Delta^{3} /|g|^{2} .
\end{aligned}
$$

We now verify, by working backwards, that this describes three straight lines intersecting at the vertices of an equilateral triangle.

For the moment, assume the points $\mathcal{S} \equiv\left\{1, e^{i 2 \pi / 3}, e^{-i 2 \pi / 3}\right\} \quad$ comprise three vertices in the complex $z$-plane. The line passing through the latter two is the set, $L \equiv\{z \mid \Re(z)=-1 / 2\}$. Written as a linear polynomial, the condition becomes,

$$
z+z^{*}+1=0 .
$$

By the rotational symmetry of the figure, if $z$ lies on $L$, then $e^{ \pm i 2 \pi / 3} z$ lies on the line passing through $\left(e^{\mp i 2 \pi / 3}, 1\right)$. Thus, the polynomial satisfied by the union of lines whose intersections populate $\mathcal{S}$ is,

$$
\left(e^{i 2 \pi / 3} z+e^{-i 2 \pi / 3} z^{*}+1\right)\left(z+z^{*}+1\right)\left(e^{-i 2 \pi / 3} z+e^{i 2 \pi / 3} z^{*}+1\right)=0 .
$$

Expanding and combining terms produces

$$
z^{3}+z^{* 3}-3 z z^{*}+1=0
$$

This must be connected with Eq.(1) by an appropriate dilation and rotation. First, multiply $z$ by $\left|a_{0}\right|$, to correct the scale, and identify $a=\left|a_{0}\right| z$.

$$
a^{3}+a^{* 3}-3 a a^{*}\left|a_{0}\right|+\left|a_{0}\right|^{3}=0
$$

Multiply by $-\Delta / 3\left|a_{0}\right|$ and rearrange terms.

$$
\frac{1}{3} \Delta \cdot\left|a_{0}\right|^{2}=H_{0}=\Delta \cdot a a^{*}-\left(\Delta / 3\left|a_{0}\right|\right)\left(a^{3}+a^{* 3}\right)
$$

Of course, Eq.(8) has been used to identify $H_{0}$. Now, substitute from Eq.(4) for $\Delta / 3\left|a_{0}\right|$,

$$
\begin{aligned}
\frac{\Delta}{3\left|a_{0}\right|} & =|g| e^{-i \Psi} e^{i 3 \varphi_{0}}=g^{*} e^{i 3 \varphi_{0}}=-i g^{*}\left(e^{-i\left(\pi / 2-\varphi_{0}\right)}\right)^{3} \\
& =|g| e^{i \Psi} e^{-i 3 \varphi_{0}}=g e^{-i 3 \varphi_{0}}=i g\left(e^{i\left(\pi / 2-\varphi_{0}\right)}\right)^{3}
\end{aligned}
$$


and rotate phase space so that $a e^{i\left(\pi / 2-\varphi_{0}\right)} \mapsto a$ to finish the demonstration.

$$
H_{0}=\Delta \cdot a^{*} a-i g a^{3}+i g^{*} a^{* 3}
$$

Notice that the rotation angle is $\pi / 2-\varphi_{0}$, not $\varphi_{0}$. (See Figure 1.)

Emittance of central region. The emittance of the central stable region is the "area" of an equilateral triangle with "radius" $\sqrt{2 I_{0}}$.

$$
\varepsilon=\frac{3 \sqrt{3}}{4}\left|\sqrt{2 I_{0}}\right|^{2}=\frac{1}{2 \sqrt{3}}|\Delta / g|^{2}
$$

For comparison, the emittance, $\varepsilon_{b}$, of a bunch in equilibrium with the linear machine and totally contained within the central stable region is the "area" of a circle whose radius is $\sqrt{I_{0} / 2}$, i.e. $\varepsilon_{b}=\pi I_{0} / 2$. (See Figure 2.) The ratio of the two is thus,

$$
\frac{\varepsilon_{b}}{\varepsilon_{c}}=\frac{\pi}{3 \sqrt{3}} \approx 0.605 .
$$

This number is useful for setting the initial parameters of the squeeze.

Step size. Because the outgoing branches of the separatrix are asymptotes, the behavior of orbits on those branches serves to estimate how all orbits will approach the septum's field region. To that end, it is convenient that the equation of motion along the separatrix can be expressed analytically. ${ }^{8}$

First, when $\Delta=0$, then on the separatrix, $a \equiv$ ire $e^{-i\left(\varphi_{0} \pm \pi / 6\right)}$ (i.e. $\left.r=\sqrt{I}\right)$, and

$$
\begin{gathered}
d a / d \theta=i d r / d \theta e^{-i\left(\varphi_{0} \pm \pi / 6\right)}=3 g^{*} a^{* 2} \\
=-3|g| e^{-i \Psi} r^{2} e^{i\left(2 \varphi_{0} \pm \pi / 3\right)} \\
\Rightarrow \quad d r / d \theta= \pm 3|g| r^{2} \\
\frac{1}{r_{1}}-\frac{1}{r_{2}}=3|g| \int_{0}^{3 \times 2 \pi} d \theta=18 \pi|g| \\
r_{2}-r_{1}=\frac{18 \pi|g| r_{1}^{2}}{1-18 \pi|g| r_{1}}
\end{gathered}
$$

If $\Delta \neq 0$, then scale by $a_{0}$ : i.e. $a \equiv a_{0} u$. On the separatrix,

$$
\begin{aligned}
d a / d \theta=a_{0} d u / d \theta & =-i \Delta a_{0} u+3 g^{*} a_{0}^{* 2} u^{* 2} \\
& =-i \Delta a_{0}\left(u-u^{* 2}\right) \\
d u / d \theta & =-i \Delta\left(u-u^{* 2}\right)
\end{aligned}
$$

Now let $u \equiv 1+r e^{i \pi / 6}$. (Note: $r$ is dimensionless in this expression. Units and scaling were absorbed by $a_{0}$.) Then, due the remarkable facts that

$$
e^{i \pi / 6}-2 e^{-i \pi / 6}=-\sqrt{3} e^{-i \pi / 3} \text { and } i e^{-i \pi / 6}=e^{i \pi / 3}
$$

we get

$$
d r / d \theta=\Delta\left(\sqrt{3} r+r^{2}\right)=\Delta \cdot r(\sqrt{3}+r)=\Delta \cdot\left[(r+\sqrt{3} / 2)^{2}-3 / 4\right]
$$

\footnotetext{
${ }^{8}$ To the best of our knowledge, this calculation was first done by Edwards. [4]
} 
After solving, the step size condition is written,

$$
r_{2}-r_{1}=\frac{r_{1}\left(r_{1}+\sqrt{3}\right)}{[\sqrt{3} /(\exp (6 \pi \sqrt{3} \Delta)-1)]-r_{1}}
$$

Working backwards to remove the scaling will reinstate the dependence of step size on $g$ and $\beta_{x}$.

Spill rate. Experimenters want beam to be extracted at a uniform rate. Realistically, that will require diagnostics and feed-forward to monitor and control the ramp profiles of the extraction circuits. Nonetheless, under a few assumptions whose validity ranges from "solid" to "highly dubious" - it is possible to write solutions in at least two simple cases. The assumptions are:

(a) Liouville's theorem, with its consequence that the density of states is a constant of the motion. Of course, if the system is truly Hamiltonian, then Liouville's theorem is assured.

(b) a bunch initially in equilibrium, so that the density function depends only on the action coordinate.

(c) an adiabatic squeeze. A fundamental theorem of Hamiltonian mechanics states that the value of action remains invariant under adiabatic changes in the system's parameters.

With those accepted, the following manipulations can go forward.

We assume a frozen distribution, $\rho(I)$, dependent only on the action coordinate, such that the number of particles remaining in the bunch at time $t$ is

$$
N(t)=\int_{0}^{I_{\max }(t)} d I \rho(I), \text { for } t \in[0, \tau] .
$$

We further assume a steady depletion to define $I_{\max }(t)$.

$$
N(t)=N(0) \cdot(1-t / \tau)
$$

Finally, Eq.(9) is used to connect the ramp profile, say $\Delta(t)$, with the largest action coordinate in the bunch.

$$
\Delta(t)=\Delta(0) \sqrt{I_{\max }(t) / I_{\max }(0)}
$$

Given a density function, $\rho(I)$, the task is to invert Eq.(11) to find $I_{\max }(t)$, which then is used to generate $\Delta(t)$. This can be carried out analytically in at least two simple cases, the details of which are trivial and left to the (hypothetical) reader:

Constant density:

$$
\rho(I)=\left\{\begin{array}{ll}
\rho_{c}, & I<I_{\max }(0) \\
0, & I_{\max }(0)<I
\end{array} \quad \Rightarrow \quad \Delta=\Delta(0) \sqrt{1-t / \tau}\right.
$$

Truncated Gaussian:

$$
\rho(I)=\left\{\begin{array}{ll}
\rho_{c} e^{-\Gamma I}, & I<I_{\max }(0) \\
0, & I_{\max }(0)<I
\end{array} \Rightarrow \Delta=\Delta(0)\left(-\frac{\ln \left(t / \tau+e^{-\Gamma I_{\max }(0)}(1-t / \tau)\right)}{\Gamma I_{\max }(0)}\right)^{1 / 2}\right.
$$

\subsection{Half integer}

The half-integer resonance exemplar is written in Eq.(43), of Appendix B, and transcribed here.

$$
H=\Delta \cdot a^{*} a+G_{4}\left(a^{*} a\right)^{2}+G_{2} a^{2}+G_{2}^{*} a^{* 2}+\cdots
$$


Here, as usual, $\Delta \equiv v_{x}+n / 2 \approx 0$. The first two terms express amplitude dependence of the tune, due to the presence of octupoles; the last two contain the resonance. Two coupling parameters are functionals of quadrupole and octupole strength distributions.

$$
\begin{aligned}
G_{2} & =\frac{1}{8 \pi} \sum_{\text {quadrupoles }} \frac{\delta B^{\prime} l}{|B \rho|} \beta_{x} e^{-i 2\left(\psi_{x}-\Delta \cdot \theta\right)} \\
G_{4} & =\frac{1}{32 \pi} \sum_{\text {octupoles }} \frac{B^{\prime \prime \prime} l}{|B \rho|} \beta_{x}^{2}
\end{aligned}
$$

In the summand for $G_{2}$, the expression " $\delta B^{\prime}$ " indicates that only the fraction of quadrupole strength not contributing to the tune must be used here. Notice that $G_{2}$ is complex, but $G_{4}$ is real. We shall examine, in sequence, the resonant orbit, separatrix, and step size of this examplar.

Resonant orbit. Hamilton's equation of motion can be written as follows.

$$
i d a / d \theta=\partial H / \partial a^{*}=\Delta \cdot a+2 G_{2}^{*} a^{*}+2 G_{4} a^{*} a^{2}
$$

The resonant orbit is thus characterized by the fixed point equation,

$$
(d a / d \theta)_{a=a_{0}}=0 \Rightarrow \Delta \cdot a_{0}+2 G_{4} a_{0}^{*} a_{0}^{2}=-2 G_{2}^{*} a_{0}^{*}
$$

Now multiply by $a_{0}^{*}$, use Eq.(29) and define the phase, $G_{2} \equiv\left|G_{2}\right| \exp (i \Psi)$, and factor out the solution $a_{0}=0$, to get

$$
\Delta+2 G_{4}\left|a_{0}\right|^{2}=2\left|G_{2}\right| e^{i\left(2 \varphi_{0}-\Psi\right)}
$$

The physical interpretation is this: the resonant orbit occurs where the amplitude-dependent tune shift arising from the octupoles reaches the half-integer stopband. The exponential must be real; i.e. $\exp \left(i\left(2 \varphi_{0}-\Psi\right)\right)= \pm 1$. These correspond to four resonant points, two stable and two unstable. Only the unstable ones are physically relevant. They are, as we shall confirm shortly,

$$
\begin{aligned}
& \left|a_{0}\right|^{2}=\frac{\operatorname{sgn}(\Delta) \cdot 2\left|G_{2}\right|-\Delta}{2 G_{4}} \\
& 2\left|G_{2}\right|<\Delta \text { and } G_{4}<0 \Rightarrow \varphi_{0}=\Psi / 2(\bmod \pi) \\
& \Delta<-2\left|G_{2}\right| \text { and } 0<G_{4} \Rightarrow \varphi_{0}=\Psi / 2+\pi / 2(\bmod \pi) \text {. }
\end{aligned}
$$

Notice that (a) the tune must lie outside the half-integer stopband, generated by quadrupole harmonic term, and (b) the signs of $G_{4}$ and $\Delta$ must be opposite, so that amplitude dependence can shift the tune onto resonance at the location of the resonant orbit.

Separatrix geometry. The value of the Hamiltonian on the separatrix is the value it assumes on the unstable resonant orbit.

$$
H_{0}=H\left(a_{0}\right)=\Delta \cdot\left|a_{0}\right|^{2}+G_{4}\left|a_{0}\right|^{4}+G_{2} a_{0}^{2}+G_{2}^{*} a_{0}^{* 2}
$$

From the resonant point equation, Eq.(17),

$$
G_{2}^{*} a_{0}^{* 2}+G_{4}\left|a_{0}\right|^{4}=-\frac{1}{2} \Delta \cdot\left|a_{0}\right|^{2}
$$


and thus,

$$
\begin{aligned}
H_{0} & =\frac{1}{2} \Delta \cdot\left|a_{0}\right|^{2}+G_{2} a_{0}^{2} \\
& =\frac{1}{2}\left|a_{0}\right|^{2}\left(\Delta-2 G_{2} e^{-i 2 \varphi_{0}}\right) \\
& =\frac{1}{2}\left|a_{0}\right|^{2}\left(\Delta-2\left|G_{2}\right| e^{-i\left(2 \varphi_{0}-\Psi\right)}\right) \\
& =\frac{1}{2}\left|a_{0}\right|^{2}\left(\Delta-2\left|G_{2}\right| e^{i\left(2 \varphi_{0}-\Psi\right)}\right) \\
& =-G_{4}\left|a_{0}\right|^{4} .
\end{aligned}
$$

where Eq.(18) has been used to get the last line.

With this result in hand, we can now establish that the separatrix consists of two intersecting circles. Let $z$ represent a complex variable, $c$ a complex number, and $r$ a real, positive number. The points in the complex plane consisting two circles centered at $\pm c$, each having radius $r$, are completely determined by the equation,

$$
\left(|z-c|-r^{2}\right) \cdot\left(|z+c|-r^{2}\right)=0 .
$$

Upon expanding and rearranging terms, this becomes the following.

$$
|z|^{4}-2 r^{2}|z|^{2}-c^{2} z^{* 2}-c^{* 2} z^{2}=-\left(r^{2}-|c|^{2}\right)^{2}
$$

For comparison, combine Eq.(14) and Eq.(20) to obtain the equation for the separatrix.

$$
G_{4}|a|^{4}+\Delta \cdot|a|^{2}+G_{2}^{*} a^{* 2}+G_{2} a^{2}=-G_{4}\left|a_{0}\right|^{4}
$$

The result is established when we identify,

$$
\begin{aligned}
r^{2} & =-\Delta / 2 G_{4}, \\
c^{* 2} & =-G_{2} / G_{4}, \text { and } \\
r^{2} & =\left|a_{0}\right|^{2}+|c|^{2} .
\end{aligned}
$$

The first two connect the geometric attributes of the circles with the control parameters of the Hamiltonian. The third is just the Pythagorean theorem, as $\left|a_{0}\right|$ is the distance from the origin to the point of intersection. Its consistency with the first two must be verified.

$$
r^{2}-|c|^{2}=-\frac{\Delta}{2 G_{4}}-\frac{\left|G_{2}\right|}{\left|G_{4}\right|}
$$

However, we rewrite,

$$
\left|G_{4}\right|=G_{4} \cdot \operatorname{sgn}\left(G_{4}\right)=-G_{4} \cdot \operatorname{sgn}(\Delta)
$$

to get,

$$
r^{2}-|c|^{2}=\frac{\operatorname{sgn}(\Delta) \cdot 2\left|G_{2}\right|-\Delta}{2 G_{4}}=\left|a_{0}\right|^{2},
$$

in agreement with Eq.(19).

Modulo a dilation, the shape of the separatrix is determined by the ratio,

$$
|c| / r=\cos (\theta / 2)=\left(2\left|G_{2} / \Delta\right|\right)^{1 / 2},
$$


which thus we shall call (at least once) the "shape parameter." Here, $\theta$ is the angle subtended by the resonant points at the center of one of the lobes. (See Figure 4.)

Emittance of central region. Emittance of the central stable region is obtained by a calculation suitable for a geometry exercise at the junior high school level. With reference to Figure 4, the area of the circular sector subtended by angle $\theta=2 \cos ^{-1}(|c| / r)$ is $\theta r^{2} / 2$. The area of the triangular piece is $r \sin (\theta / 2) \cdot r \cos (\theta / 2)=r^{2} \sin (\theta) / 2$. Subtracting one from the other provides the area of the shaded cap. Keeping in mind that "emittance" is double the value of "area" in these coordinates, we have

$$
\varepsilon_{c}=2 r^{2}(\theta-\sin \theta), \quad \theta \in[0, \pi],
$$

for the emittance of the central region.

If a circle now be inscribed, as shown on the right of Figure 4, representing the initial bunch, the ratio of its emittance to that of the central region is,

$$
\frac{\varepsilon_{b}}{\varepsilon_{c}}=\frac{\pi(r-|c|)^{2}}{r^{2}(\theta-\sin \theta)}=\frac{\pi(1-\cos (\theta / 2))^{2}}{\theta-\sin \theta}
$$

Step size. As the separatrix is comprised of two circles, along it we can parametrize,

$$
a=c+r e^{-i \eta},
$$

where the sign in the exponential is chosen to correspond to a monotonically increasing phase when $\Delta<0$. (See the right hand side of Figure 4.) Trajectories along the separatrix are determined by the differential equation,

$$
\frac{d \eta}{d \theta}=\frac{1}{r} e^{i \eta}\left(i \frac{d a}{d \theta}\right)
$$

To fill this in, one can use Eq.(21) to specialize Eq.(16) to motion along the separatrix.

$$
\text { on the separatrix : } i a^{*} \frac{d a}{d \theta}=\Delta \cdot\left(\left|a_{0}\right|^{2}-|a|^{2}\right)+2 G_{2} \cdot\left(a_{0}^{2}-a^{2}\right)
$$

In general, Eq.(26) is solved numerically, but a simplification occurs when the two lobes of the separatrix are tangent: i.e. the emittance of the central stable region is zero. This corresponds to the conditions,

$$
|\Delta|=2\left|G_{2}\right|, \quad\left|a_{0}\right|=0, \quad \text { and } c=i r e^{-i \Psi / 2} .
$$

From Eq.(20) we get an immediate consequence,

$$
H_{0}=0 \Rightarrow G_{2}^{*} a^{* 2}+G_{4}\left(a^{*} a\right)^{2}=-\Delta \cdot a^{*} a-G_{2} a^{2},
$$

which, substituted into Eq.(16) and Eq.(25), gives us the simpler expressions,

$$
\begin{aligned}
\text { on the separatrix : } & a=i r e^{-i \Psi / 2}\left(1-i e^{-i(\eta-\Psi / 2)}\right) \\
& i d a / d \theta=-2\left|G_{2}\right| a\left(\operatorname{sgn}(\Delta)+e^{i \Psi} a / a^{*}\right) \\
\text { and therefore : } & \frac{d \eta}{d \theta}=-2\left|G_{2}\right|\left(e^{i \eta} a / r\right)\left(\operatorname{sgn}(\Delta)+e^{i \Psi} a / a^{*}\right) .
\end{aligned}
$$


Employing a little algebra on Eq.(27) reveals that

$$
\begin{aligned}
e^{i \eta} a / r & =1+i e^{i(\eta-\Psi / 2)} \\
\text { and }-1+e^{i \Psi} a / a^{*} & =-2 \cdot \frac{1-\sin (\eta-\Psi / 2)}{1+i e^{i(\eta-\Psi / 2)}}
\end{aligned}
$$

on the separatrix. Putting these pieces together lets us rewrite Eq.(28) as follows.

$$
\frac{d \eta}{d \theta}=4\left|G_{2}\right|(1-\sin (\eta-\Psi / 2))
$$

The reduction to quadrature and its solution is done easily now.

$$
\begin{aligned}
4\left|G_{2}\right| \int_{0}^{2 \times 2 \pi} d \theta=16 \pi\left|G_{2}\right| & =\int_{\eta_{0}}^{\eta_{0}+\delta \eta} \frac{d \eta}{1-\sin (\eta-\Psi / 2)} \\
& =\frac{1}{2} \int_{\eta_{0}}^{\eta_{0}+\delta \eta} \frac{d \eta}{\cos ^{2}(\eta / 2-\Psi / 4+\pi / 4)} \\
& =\left.\tan (\eta / 2-\Psi / 4+\pi / 4)\right|_{\eta_{0}} ^{\eta_{0}+\delta \eta}
\end{aligned}
$$

This is then solved for the quantity $\delta \eta$, which becomes the step size when (correctly) projected onto the horizontal axis. $\delta \eta$ will be small when the orbit begins near the resonant point, i.e. when $\eta_{0}-\Psi / 2 \approx \pi / 2$. In that case,

$$
16 \pi\left|G_{2}\right| \approx \frac{\delta \eta}{1-\sin \left(\eta_{0}-\Psi / 2\right)} \Rightarrow \delta \eta \approx 16 \pi\left|G_{2}\right| \cdot\left(1-\sin \left(\eta_{0}-\Psi / 2\right)\right)
$$

is a good approximation. (See Figure 5.)

Spill rate. While there would be some satisfaction in writing analytic expressions similar to Eq.(12) and Eq.(13) for the tune profiles associated with a constant spill rate, we cannot. Starting from Eq.(23) to effect the inversion is much more complicated than from Eq.(9). We shall leave this to numerical calculations.

\section{Numerical computations}

A small set of numerical calculations were carried out, primarily to validate the exemplars within the context of tracking programs, and, mutatis mutandi, to test the proper working of the programs. The models used were based on "The Fermilab Antiproton Source Design Report" [10] and written into MAD V.8 description files (i.e. ".lat" files). More accurate descriptions of the Debuncher and Accumulator rings as they now exist are stored in the Accelerator Division's Lattice Repository ${ }^{9}[11]$ but were not used because (a) they are written in Optim format, for which we have no parsers other than the Optim program itself, (b) for purposes of design and understanding it is best to start from a "perfect," symmetric machine, and (c) the rings will, in any case, undergo changes required by the mu2e experiment. In particular, the dihedral symmetry of the Debuncher will be broken by (as yet unspecified) changes being designed for one of its straight sections. We shall not incorporate those changes here but assume they will not change the linear lattice functions in the other two sides of the ring.

\footnotetext{
${ }^{9}$ The repository is still available at http://lattices.fnal.gov/ .
} 
Both MAD itself and CHEF's [12, 13, 14] parsers can interpret lattice descriptions in MAD v.8 and Xsif format, of which we chose the former for writing the files, which have been included in two appendices for completeness. ${ }^{10}$ To the base lattice for the Debuncher were added control elements to excite either a third-integer or a half-integer resonance. The configurations and the calculations carried out on them are described in the two subsections below.

NOTE: An important feature common to both is that all extraction control elements, including the lambertson and septum, were added within the zero-dispersion portions of two straight sections. In addition, the Debuncher's chromaticity was set (near) to zero using its pre-existing chromaticity sextupoles; a scan of chromaticity as a function of $\Delta p / p$ in our model is shown in Figure 6. ${ }^{11}$ Taken together, these two circumstances eliminate first order chromatic effects on the extraction process.

\subsection{Third integer computations}

\subsubsection{Lattice modifications}

Tune control throughout the squeeze will be handled by the quadrupoles in two of the (zero-dispersion) straight sections. Thus, there will be no need for new quadrupoles to be added to the Debuncher. However, six "harmonic" sextupoles must be added to the base lattice to excite the third integer resonance. Their locations are shown schematically in Figure 7 and projectively in Figure 8; the latter also illustrates locations of the septum and lambertson. ${ }^{12}$ More to the point, the six phasors that appear in the resonance sum, $g$, of Eq.(2) are shown on the left hand side of Figure 9. By reversing the polarity of the "-2" locations relative to the "-1" and "-3", we see that sextupoles in the " $20-x$ " and " 50 -x" locations form almost perfectly orthogonal circuits. (That they are not perfect is not important, as pointed out in Appendix C.) With them we can adjust both amplitude and phase of $g$ as desired.

\subsubsection{Computations vs. exemplar}

Three real parameters enter into the exemplar:

$G_{3}$ : real and imaginary parts (or amplitude and phase) of N/3 harmonic sextupole coupling constant

$\Delta:$ difference between the base and resonant tunes

Further, the phase of $G_{3}$ determines the orientation of the separatrix, while its scale is determined by the ratio, $\left|\Delta / G_{3}\right|$, according to Equations (5) through (7). For the purpose of setting up simulations, we treat $G_{3}$ and $\Delta$ as derived from the quantities:

$\varepsilon_{b}:$ initial transverse emittance of the bunch

$\varphi_{0}:$ angle of orientation

More properly, the separatrix must be oriented according to the phase advance between the septum and the lambertson, as indicated in Figure 21. The integrated electric field of the septum is then fixed by the requirement of hitting the target region shown in the figure.

Squeezing the separatrix is accomplished either in the limit $\Delta \rightarrow 0$ or $G_{3} \rightarrow \infty$. Of course, the latter is impractical, so the former will be done. Space charge (not addressed here) will reduce and spread the tunes. Extracting particles in the expected sequence - larger amplitudes first; the core last - requires that $\Delta<0$ be used.

\footnotetext{
${ }^{10}$ They are also available from Fermilab's mu2e document database.

${ }^{11}$ Of course, non-zero chromaticity may prove necessary, in which case it later will have to be taken into account.

${ }^{12}$ In the interest of full disclosure, the MAD file positions the sextupoles in sectors 50 and 20, with 40 and 30 intervening, and puts the septum and lambertson in sector 10. The labels in the Figure 7 agree with our current usage of the straight section in sectors 20 and 30 for extraction. Because of symmetry, this renaming makes no difference.
} 
Ramping quadrupoles to effect the squeeze will change not only the tune but the relative phases between the sextupoles, affecting the value of $g$. The ranges of phasors shown in the left hand side of Figure 9, indicate the extent over which each will vary. The important point here is that they are small, which means that the value of $g$ will remain reasonably constant if harmonic sextupole strengths are fixed throughout extraction. They need not track the quadrupoles. For comparison, the right hand side of Figure 9 illustrates the variation in $\psi$, rather than $\psi-v \theta$.

Resonant orbit and separatrix. The first issue to be settled is whether the exemplar adequately locates the resonant orbit, without further adjustments from higher orders of perturbation theory. To that end, a comparison was made between the solution expressed in Equations (5) through (7) and direct, exact computations of the period three orbit of the modified Debuncher model. The result is shown in Figure 10. The exact orbit was computed using the toolkit available in CHEF's libraries $[12,13,14]$. Agreement seems adequate for a fairly large range in horizontal tune, approximately $9.64<v_{x}<9.69$.

However, taken by itself, this "agreement" is misleading. Figure 11 shows a more complete comparison between the exact separatrix, obtained by tracking nearby orbits, with that of the exemplar for $v_{x} \in[9.62,9.66]$. The controls were fixed so that the central stable region could encompass a circle of invariant emittance $20 \pi \mathrm{mm}$ - $\mathrm{mr}$. That is, the emittance of the exemplar's triangle was fixed at $(3 \sqrt{3} / \pi) \cdot 20 \pi / \beta \gamma \approx 11 \mathrm{~mm}-\mathrm{mr}$, regardless of the tune by using the harmonic sextupoles to track the value of $g$. (See Equations (9) and (10).) To accomplish this, the magnitude of each sextupole's integrated strength changed linearly with tune from $\approx 15 \mathrm{~T} / \mathrm{m}$ at $v_{x}=9.66$ to $\approx 107 \mathrm{~T} / \mathrm{m}$ at $v_{x}=9.62$. Thus, we examine here the integrity of the separatrix at the start of the squeeze. With reference to Figure 11, the small distortions in the triangular shape are unimportant but not the behavior of the outgoing branches, which deviate significantly for $v_{x}<9.64$ and are completely broken at $v \approx 9.62$ or below. Given this configuration of magnets, This bounds the range of tunes for the squeeze to $9.64 \leq v_{x} \leq 29 / 3$. We note in passing that, nearer to $v_{x}=9.64$, one outgoing branch is better matched to the exemplar than the other two. ${ }^{13}$ This circumstance merits some attention, but not here and not now.

Approach ramp. To be realistic for just a moment, the ramp profile of the tune (control quadrupoles) controlling the spill will be established experimentally and experientially early in the course of the mu2e experiment. Nonetheless, for whatever it's worth, the profiles suggested by Equations (12) and (13) are shown in Figure 3. The top, dashed curve corresponds to the limiting behavior for a flat distribution, i.e. Eq.(12); the three lower curves assume truncated Gaussians with $90 \%, 95 \%$, and $99 \%$ of the initial emittance enclosed at the start. ${ }^{14}$

\subsubsection{Extraction simulations}

A short program was written to simulate (third-integer) extraction, in the absence of space charge, using the sextupole and quadrupole control elements discussed above. Using the tools available in CHEF's libraries, the program

(a) reads and interprets the (MAD v.8) design lattice file and, from it, builds a software model of the Debuncher.

(b) groups the quadrupoles in the zero-dispersion regions of two of the straight sections, effectively attaching them to "circuits" so that their strengths are controlled as one.

(c) inserts sextupoles, septum and lambertson elements in locations as specified above.

(d) attaches sextupoles to two "circuits," also as specified above.

(e) using initial conditions of tune and emittance specified as command line arguments, sets the strengths of the sextupoles so the separatrix will encompass the initial distribution.

(f) populates a "bunch" of protons that would be in equilibrium were the sextupoles turned off. (This could be done using a variety of distributions, but only a uniform density was tried.)

\footnotetext{
${ }^{13}$ By a happy accident, this is the one to be used for extraction.

${ }^{14}$ I (LM) refuse to comment on the surprising result that the $90 \%$ and $95 \%$ curves are closer than than the $95 \%$ and $99 \%$ ones.
} 
(g) performs a $16 \mathrm{msec}$ squeeze to extract the protons by ramping the tune-control quadrupoles.

Simulations were carried for bunches consisting of 64, to debug the program, to 4096 protons. While the ramp profile could be anything - e.g. as specified by Eq.(12) - only a linear profile was used. For these runs, chromaticity was essentially zero and no aperture restrictions were introduced into the ring, so that momentum deviations had negligible effect.

Figure 12 shows a typical result, plotting the fractional population of the bunch as a function of time. Unless care is employed to tailor the ramp - e.g. as suggested by Equations (12) or (13), or at least beginning the ramp carefully so that the bunch starts well within the separatrix and has time to adjust to it - as much as 10-20\% of the bunch can be depleted very rapidly, within the first score of turns. The severity is greatest for a uniform density, as otherwise fewer particles exist at the bunch's fringes. After the separatrix has had time to reshape the distribution, the spill rate remains reasonably consant over about half of the time interval, at which point $\approx 80 \%$ of the bunch has been removed. Without adjusting the ramp, the rate is reduced while the final $\approx 20 \%$ is extracted.

Of course, this initial burst of extracted particles would be unacceptable, emphasizing the need to design a ramp profile that avoids it. ${ }^{15}$ Undoubtedly, final adjustments will be based on operational experience.

A snapshot of states in horizontal phase space is shown in Figure $13 .{ }^{16}$ It was taken at the $600^{\text {th }}$ turn, approximately $1.1 \mathrm{msec}$ into the ramp, well after the initial transient, after the bunch distribution has accomodated itself to the shape of the separatrix. The bunch initially contained 4096 protons, of which 3022 remained to be immortalized in this picture.

Because mu2e requires large bunches intensities, space charge is expected to affect extraction significantly. The ingredients of the simple simulations reported here have been handed over to others ${ }^{17}$ for inclusion of space charge effects. Its only influence on our preliminary work was that - because space charge reduces tunes, producing a tune spread with maximum shift within the core - it was (finally) decided to approach the resonance from below. Doing so shifts the core away from the resonance, assuring that particles will be extracted from the bunch in the expected order: i.e. larger amplitudes first.

\subsection{Half integer computations}

\subsubsection{Lattice modifications}

By the time of writing, fewer computations were carried out for the half-integer resonance for a number of reasons, two of which are: (1) the third-integer was initially given almost exclusive priority and (2) five or six weeks were wasted chasing a rather unworkable approach for a half-integer configuration. To get that out of the way first, its principal features included:

(a) a global shifting of the Debuncher's tune to near 19/2.

(b) using four already existing focusing quadrupoles in the center of one straight section to generate the $G_{2}$ harmonic term. (c) placing three (thin) octupoles symmetrically at the centers of the straight sections to generate the zeroth harmonic $G_{4}$ term.

(d) effecting the squeeze by altering the strengths of the harmonic quadrupoles, increasing the stopband width, effectively $G_{2}$, while keeping the tune constant.

In principle, this might have been made to work; in practice, it seemed too sensitive to phase shifts induced by the quadrupole harmonic circuits. It is important that the second and fourth harmonic octupolar principal terms vanish. The

\footnotetext{
${ }^{15} \mathrm{Jim}$ Amundson, who is including space charge in his simulations, approached the initial stage of the ramp somewhat more carefully and, without much effort, reduced the spill to $0.5 \%$. This is much smaller but still too large. That number is not a lower bound and will be improved.

${ }^{16}$ I (LM) think, but will not guarantee, that this "snapshot" was taken just upstream of the septum.

${ }^{17}$ That is, to Jim Amundson.
} 
phasor diagrams in Figure 14 demonstrate that one trivial way to do this is to take advantage of the ring's symmetry and place octupoles at the centers of the three straight sections. However, the harmonic quadrupole circuit breaks that symmetry, inducing phase errors between the octupoles that end up distorting the separatrix badly.

A more sensible approach has been taken recently, consisting of the following steps.

(a) bring the horizontal tune to exactly 19/2 by locally adjusting the phase advances within each cell of the straight sections to be $60^{\circ}$, as in the arcs.

(b) insert new quadrupole correctors at eight locations. The locations are chosen so that, when powered symmetrically, the first order tune variation vanishes and the quads form orthogonal families with respect to the half-integer phasors. These eight will be used to control $G_{2}$.

(c) tune the circuits by observing the variation in tune with excitation and setting them where the minima occur.

The locations of the eight harmonic quadrupoles that would be installed in the Debuncher are shown in Figure 15, with the two families identified and labelled. The tuning step taken in (c) is now standard practice ${ }^{18}$ to compensate for quadrupole field errors throughout the ring. In the absence of errors, the tune dependence on excitation of the two quad circuits is shown on the left-side of Figure 16. (The bare tune has been set to 9.485.) Minima occur at zero excitation, as expected; that is, the harmonic circuits do not affect the tune linearly. After field errors are introduced randomly throughout the Debuncher's quadrupoles, the tune dependence is shown on the right-hand side of Figure 16. The minima have shifted, and these excitations must be used as the "base" or "zero point" upon which to build the driving term, $G_{2}$.

Evidence that this global strategy adequately stabilizes phases locally is offered by the curves in Figure 17, which show $\Delta \beta / \beta$ before (red) and after (blue) the harmonic circuits have been tuned. The lattice functions themselves are displayed in Figure 18.

\subsubsection{Computations vs. exemplar}

Four parameters enter into the exemplar:

$G_{4}:$ zeroth harmonic octupoles' coupling constant

$G_{2}$ : real and imaginary parts (or amplitude and phase) of N/2 harmonic quadrupoles' coupling constant

$\Delta:$ difference between the base and resonant tunes

For the purposes of setting up simulations, we treat $G_{4}$ and $\left|G_{2}\right|$ as derived from the more fundamental quantities:

$|c| / r$ : shape parameter, equivalent to $\theta=2 \cos ^{-1}(|c| / r)$

$\varepsilon_{b} \quad$ : initial transverse emittance of the bunch

$\theta$ is the angle subtended from a lobe's center by their points of intersection, the resonant orbit.

We shall adopt the set of four parameters, $\mathcal{P} \equiv\left\{\varepsilon_{b}, \Delta,|c| / r\right.$ (or $\left.\left.\theta\right), \Psi\right\}$, as determining the exemplar of the simulation. From them, $G_{2}$ and $G_{4}$ are derived as follows.

$$
\begin{aligned}
\left|G_{2}\right| & =|\Delta / 2|(|c| / r)^{2}, \text { and, of course, } G_{2}=\left|G_{2}\right| e^{i \Psi} \\
G_{4} & =-\Delta \cdot\left(\varepsilon_{b} / \pi\right)^{-1}(1-|c| / r)^{2}
\end{aligned}
$$

\footnotetext{
${ }^{18}$ One of the authors $(\mathrm{JJ})$ has had operational experience with half-integer extraction from the Main Injector; the other (LM) tried to compensate by spending several hours speaking with him and with others who controlled extraction from the Tevatron. Step (c) is standard in the Main Injector and became standard in the Tevatron, though at some point - prior to the studies reported in References [15] and [16], which does not necessarily imply causality - Tevatron extraction was finely tuned by detecting and minimizing particle losses. [17] We expect that should be done for mu2e as well. The complete history of resonant extraction at Fermilab is worth documenting, but not here and not now.
} 
These are obtained by combining the pieces of Eq.(22) with the observation that, when the bunch exactly fits into the central region, then it must be that $\varepsilon_{b} / 2=\pi(r-|c|)^{2}$. Note in passing that, as one would expect, the quadrupole constant is connected with shape only, while scale is determined by the octupoles.

Of course, $\Psi$ must not be chosen randomly. As with the third integer, it contols the orientation of the separatrix, so its value will depend on the phase advance between the septum and the lambertson. (E.g. see Figure 21, for the third-integer.) Further, the septum offset will be influenced by the separatrix as well as the step size of its asymptotic orbits.

Resonant orbit and separatrix. As with the third integer, we must first examine how well the exemplar, with no adjustments from higher orders of perturbation theory, can describe the actual resonant orbit and its separatrix. Figure 19 shows two representative snapshots, taken at $v_{x}=9.46$ and $v_{x}=9.49$. Notice the "interwoven figure eight" sub-separatrices appearing within the major lobes at the lower tune. (In connection with this, see also Figure 22 and footnote 31 from the discussion in Appendix B.) The red circles comprise the exemplar's separatrix, while orbits from tracking are shown in black. As before, and as expected, the closer one gets to the resonant tune, the better. Even so, achieving a better match to the orientation would require additional quadrupole tuning.

Many such separatrices were constructed by examining a variety of quadrupole arrangements and settings. The zoological collection of pictures will not be displayed here. Suffice it to say that hours of staring at them resulted in an increased awareness of the ease with which a half-integer separatrix could be distorted by such deviations. The presumed mechanism is that variations in phase advance break symmetry and hinder the suppression of unwanted second and fourth order octupole harmonic terms. ${ }^{19}$ The "geometry" and even topology of the separatrix is sensitive to such terms; e.g. see References [3] and [5].

\subsubsection{Extraction simulations}

Unlike the third-integer, there are (at least) three inequivalent ways to perform the squeeze:

(a) by fixing $G_{2}$ and $G_{4}$ and reducing $\Delta$, the separation between the two lobes remains fixed while their radii shrink,

(b) by proportionately reducing the magnitudes of $\Delta$ and $G_{4}$, so that the ratio $\Delta / G_{4}$ remains constant, the lobes' radii are fixed while their separation increases, or

(c) by fixing $\Delta$ and $G_{4}$ while varying $G_{2}$, producing the same effect as method (b),

until the squeeze is completed by reaching the point of tangency. For the record, method (c) was used in the Tevatron and is still used in the Main Injector.

It may be worth mentioning, as a historical sidebar, that second harmonic octupoles were installed in the Tevatron but never powered, after it was decided to use only the zeroth harmonic distribution. ${ }^{20}$ In the Main Injector, zeroth harmonic octupoles were installed, to excite the average term but, as it turned out, neither needed nor powered due to the unexpectedly large octupole content of the Main Ring quadrupoles.

As of this writing, while a considerable number of hours have been spent staring at its separatrices, no extraction simulations have been carried out on the half-integer resonance similar to those done for the third-integer. (See Figure 20.) When and if they are, results will be included in a revision. Until that happy day, review the early simulations carried out by Harrison [19, 20, 21, 22] and the later studies of Marriner and Martens [15, 16].

\footnotetext{
${ }^{19}$ See the discussion of "Neglected principal terms" on page 24. The global quadrupole tuning strategy alluded to in the previous section may improve matters. This will be studied further in the future.

${ }^{20}$ According to Lee Teng [3], Sho Ohnuma first suggested using zeroth harmonic octupoles exclusively. Nothing of that observation was recorded apart from Teng's footnote; Ohnuma himself has no memory of it. [18]
} 


\section{Summary}

We have modeled the rings of the antiproton source based on descriptions in their design reports ${ }^{21}$ and transcribed the models into two MAD v.8 description files which are included in appendices. These are not final, as one side of the Debuncher is even now being redesigned to accommodate extraction. The new side will no doubt break the ring's (dihedral) symmetry, presumably without altering lattice functions in the other two sides. Anticipating these changes, we confined the resonance control elements to the dispersion free parts of straight sections within two sides of the Debuncher. The third will contain the (electric) septum and (magnetic) lambertson devices for extracting the beam.

Two possible resonances are under consideration: a third-integer, $v_{x}=29 / 3$, advanced by the original mu2e proposal [1], and a half-integer, $v_{x}=19 / 2$, suggested more recently, during the series of working meetings convened by Mike Syphers. Neither has (yet) been shown to be unmanageable, but each possesses its own characteristics.

The two resonances will be controlled in different ways: the half-integer, by using quadrupole harmonic circuits to enlarge the stopband; the third-integer, by using the a tune-control circuit to move the bare tune onto the resonance, thereby reducing the emittance enclosed by the central stable region to zero. ${ }^{22}$ The half-integer resonance can take advantage of a stopband width to extract the entire beam. The third-integer possesses no such width - that is, to zero the central stable region, the machine must operate precisely at the resonant tune - and is thus more sensitive to small power supply ripple. [24] Thus, using the third-integer would require an extra step (and additional hardware) to remove residual beam after extraction.

On the other hand, the separatrix of the half-integer resonance is more easily distorted than that of the third-integer. This is not unexpected, as quadrupoles are used not only to control the tune but to drive the resonance. In addition, a half-integer resonance is necessarily also a quarter-integer resonance. ${ }^{23}$ Any manipulations with quadrupoles will have to be done carefully so as to maintain the phase advances required to zero out principal octupole driving terms not included in the exemplar. ${ }^{24}$ This is done most easily by maintaining symmetry rigorously, but as already mentioned, that will not be an option. In any case, quadrupole circuits will have to be tuned operationally to their correct working points (e.g. see Figure 17).

In general, the third-integer separatrix should be more "robust": less easily distorted by small perturbations in phase advances and requiring less operational tuning than the half-integer. Because its resonance phasors are reasonably stable during extraction (see Figure 14) and there is no danger of exciting an unwanted principal resonance in the horizontal plane, tracking the tune circuit with the sextupoles should not be necessary. The exit branch of the separatrix is itself a simpler affair, being a "straight line" in normalized phase space ${ }^{25}$ and therefore matched to the target region of the lambertson (see Figure 21). Its already mentioned disadvantage is the extra "clearing" step that would be necessary.

All of which is not necessarily meant to "sell" the third-integer nor to suggest that the choice has been made already. It has not. Both possibilities will be pursued further.

Space charge will reduce the protons' tunes, especially in the core, where the electric field changes most rapidly. This suggests we should approach the resonance from below, as that would move the core away from the resonance, assuring that particles will be depopulated in the order expected. We further assume that the septum and lambertson will kick the beam to the outside. This was, for a time, an open question which now seems to have been settled. (By a happy

\footnotetext{
${ }^{21}$ Three seem to have been distributed. The first, "The Fermilab Antiproton Source Design Report," [10] was written in February, 1982; the next two, seemingly identical, bear the same title, "Design Report Tevatron 1 Project," [23] and are dated September, 1983 (blue cover) and September, 1984 (pink cover).

${ }^{22}$ The alternative would be to increase the sextupole strengths, which would reduce the emittance but not zero it.

${ }^{23}$ Whereas a third-integer is not an integer. See the discussion of "Neglected principal terms" on page 24.

${ }^{24}$ The Tevatron had the advantage of being rather large, with an abundance of symmetric locations in which to place octupoles. Confining ourselves to (zero-dispersion parts of) two straight sections in the Debuncher makes it more challenging to establish the zeroth harmonic exclusively and stably throughout the squeeze.

${ }^{25}$ In the approximate range $9.64<v_{x}<9.67$; below this, these branches have disintegrated.
} 
circumstance, this works well with approaching the resonance from below. Because protons rotate clockwise, as seen from above, this corresponds to the negative $x$ direction in transverse phase space.)

We emphasize one last time, the work reported here barely begins the effort that will be required to design, validate and perform resonant extraction from the Debuncher. These preliminary notes were compiled for easy future reference. Our intent is that they be picked up some day by a young, intelligent graduate student with the motivation, vitality, and time to give this problem sustained and undivided attention. We too will continue to work the problem, as permitted by time, obligations, and circumstance. Additional notes, building upon this one but shorter, may be written to mark future progress. We hope that parts of this memo and of those hypothetical others will find their way into the future $\mathrm{CD}<\mathrm{N}>$ documents required for the experiment to proceed. Mu2e seems likely to be approved and realized following close upon the Tevatron era. Its success will contribute to Fermilab's continued survival, even if - though we hope for more "success" turns out to mean measuring with great accuracy that nothing revolutionary is happening. 


\section{References}

[1] R. M. Carey and et al. Proposal to search for $\mu^{-} n \rightarrow e^{-} n$ with a single event sensitivity below $10^{-16}$. Technical report, Fermilab, October 2008.

[2] Robert J. Kreyche. First Philosophy; An Introductory Text in Metaphysics. Holt, Rinehard and Winston, New York NY, Chicago IL, 1964. Library of Congress 59-8708.

[3] L. C. Teng. Half integral resonant extraction from the main ring. Technical Memo FERMILAB-TM-375 (0402), Fermilab, June 1972.

[4] D. A. Edwards. Comparison of half integer and third integer extraction for the energy doubler. Technical Report FERMILAB-TM-842 (UPC 034), Fermilab, December 1978.

[5] Leo Michelotti. Catastrophe and maxwell surfaces of the half integer resonance excited by quadrupoles and octupoles. Fermi Note 393, Fermilab, 1983. http://lss.fnal.gov/archive/test-fn/0000/fermilab-fn-0393.shtml.

[6] John A. Johnstone. A simplified analysis of resonant extraction at the main injector. Technical Report MI-91, Fermilab, September 3, 1993. Main Injector Note.

[7] Shoroku Ohnuma. Tolerance Requirements on $720 \mathrm{~Hz}$ Ripples in the Main Quadrupole Current During Resonance Extractions. Technical report, Fermilab, 1972. Fermilab-TM-377 (0510).

[8] Leo Michelotti. Intermediate Classical Dynamics with Applications to Beam Physics. John Wiley \& Sons, Inc., New York, 1995.

[9] Etienne Forest. Beam Dynamics: A New Attitude and Framework. CRC Press, New York, 1998.

[10] The fermilab antiproton source design report. Technical report, Fermilab, February, 1982. (Revised February 26, 2982).

[11] Jean-François Ostiguy, Michael Kriss, Michele McCusker-Whiting, and Leo Michelotti. The fermilab lattice information repository. In Presented at 2005 Particle Accelerator Conference, 2005. (FPAT007) May 16-20, 2005. Knoxville, Tennessee.

[12] Leo Michelotti and Jean-François Ostiguy. Chef: An interactive program for accelerator optics calculations. In Proceedings of 2005 Particle Accelerator Conference, 2005. (FPAT006) May 16-20, 2005. Knoxville, Tennessee. http://accelconf.web.cern.ch/AccelConf/p05/PAPERS/FPAT006.PDF.

[13] Leo Michelotti and Jean-François Ostiguy. Chef: a framework for accelerator optics and simulation. In Proceedings of 9th International Computational Accelerator Physics Conference, 2006. (ICAP 2006), Chamonix Mont-Blanc, France, October 2-6, 2006. TUAPMP02 http://bel.gsi.de/icap2006/PAPERS/TUAPMP02.PDF FERMILAB-CONF-06-373-AD http://www.slac.stanford.edu/spires/find/hep/www?r=FERMILAB-CONF-06-373-AD.

[14] Jean-François Ostiguy and Leo Michelotti. Recent improvements to chef, a framework for accelerator computations. In Presented at 2009 Particle Accelerator Conference, 2009. PAC 09. FERMILAB-CONF-09-157-APC. May 4-8, 2009. Vancouver, BC, Canada.

[15] J. Marriner and M. Martens. Measurement of tune spread in the tevatron versus octupole strength. Technical report, Fermilab, 1996. FERMILAB-EXP-199. 
[16] M. A. Martens, J. Marriner, and J. Holt. Measurement of tevatron extraction parameters and comparison of model to measurements. In Proceedings of 1997 Particle Accelerator Converence. their Diagnosis and Correction (v.1, p.72). Vancouver, B.C., Canada. May 12-16, 1997. IEEE, 1998. Session: High-Energy Hadron Accelerators and Colliders. ID 2B007. Available at http://accelconf.web.cern.ch/AccelConf/pac97/papers/pdf/2B007.PDF.

[17] Linda Spentzouris. Private communication.

[18] Shoroku Ohnuma. Private communication.

[19] Michael Harrison. Proposed Main Ring Extraction Upgrade. Technical report, Fermilab, 1978. FERMILAB-TM-0832.

[20] Michael Harrison. Monte Carlo studies on Tevatron extraction. Technical report, Fermilab, 1979. FERMILAB-UPC-115.

[21] M. Harrison. Extraction II: Local orbit bumps, extracted beam phase space and an initial look at fast resonant extraction. Technical report, Fermilab, 1979. FERMILAB-UPC-027.

[22] Michael Harrison. Extraction III: Fast resonant extraction. Technical report, Fermilab, 1979. FERMILAB-UPC-087.

[23] Design report tevatron 1 project. Technical report, Fermilab, September, 1983 and September, 1984.

[24] Kevin Brown. Private communication.

[25] Leo Michelotti. Theory and praxis of map analysis in chef; part 1: Linear normal form. Technical Report FN-0826-CD, Fermilab, October 2008.

[26] Etienne Forest and John Irwin. Single resonance theory with maps. In W. Scandale and G. Turchetti, editors, Nonlinear Problems in Future Particle Accelerators. World Scientific, 1991. Workshop Proceedings. Capri, Italy. April 19-25, 1990. 


\section{A Conceptual schematic of third-integer extraction.}

This section explains the sequence of sketches in Figure 21 that illustrate the concept of third-integer resonance extraction. It was written primarily for someone completely new to the subject. Taken one frame at a time, with reference to Figure 21:

(a) Phase space is viewed at (just upstream of) the lambertson. The blue region corresponds to the physical region containing the magnetic field that will effect the final extraction of particles from the Debuncher. ${ }^{26}$ The darker green region is the subset encompassing the permitted region in angle as well as position, which is a small neighborhood of $x^{\prime} \approx 0$. As $\alpha \neq 0$, this regions is not parallel to the $x$-axis in normalized phase space.

(b) Phase space is here viewed at (just upstream of) the electrostatic septum, which is upstream of the lambertson. As the coordinates are (still) normalized, the target region shown in the previous sketch is simply rotated by the betatron phase angle between the two locations. The orange region now represents the region of the lambertson containing an electric field to kick particles onto new orbits.

(c) A third-integer separatrix is imposed by the harmonic sextupoles. We assume the base (zero-amplitude) tune is below resonance, so that orbits rotate counter-clockwise inside the boundary. Particles at or near the boundary escape on the outgoing arms, identified with arrows.

(d) When (and if) a particle reaches the septum's field region, it is kicked. The electric field is fixed so that, at the beginning of the squeeze, particles are kicked into the "lower" portion of the target region.

(e) As the squeeze progresses, the triangular separatrix shrinks. If the electric field remains at a fixed value, the kicked particle's will move "upward" toward the "higher" portion of the target region. (Remember that the negative $x$-axis is toward the outside of the ring. Extraction begins with particles kicked to the outer edge of the lambertson and ends with them kicked to its inner edge.) At the same time, the track does not follow what would be a line of constant $x^{\prime}$ at the lambertson. Thus, the incident angle at the lambertson will change throughout the squeeze, as was, in fact, observed in the Tevatron.

(f) What is shown in this frame will not happen. If the initial emittance were so large that the range between the lambertson's outer and inner edges was too small to encompass the entire squeeze, the separatrix would have to be rotated during the squeeze in order to finish it. To repeat, that should not be necessary.

Extraction using a half-integer resonance proceeds similarly. The major difference arises from separatrix geometry: two intersecting circles rather than an equilateral triangle, with the consequence that the outgoing arm is curved, not matching the target region. In addition, the squeeze does not correspond to a simple dilation, so the separatrix's shape is not an invariant, as is the case with the third-integer. If it is done in the Debuncher as it was in the Tevatron, the circles will separate while maintaining their radii.

\footnotetext{
${ }^{26}$ Ignore the red region; it was originally intended to convey a notion that part of the lambertson's field should be avoided.
} 


\section{B Fundamentals from linear dynamics and perturbation theory}

This appendix contains a necessarily telegraphic description of the sequence of steps which filter the two principal resonance exemplars used in this memo from a periodic Hamiltonian whose nonlinearity is expressed as a longitudinal vector potential. These steps are well known, so the description is brief, primarily intended to anchor the notation and verify the coupling constants. For our immediate purposes, you may gloss over deep questions and treat them as incantations. ${ }^{27}$ We confine ourselves to two-dimensional (horizontal) phase space, but everything can be extended naturally to higher dimensions without essential changes in the formalism.

Linear normal form coordinates: The linear normal coordinates and their relationships with (linear) action-angle coordinates and (linear) Courant-Snyder lattice functions are as follows. [8, 25] Let $\mathbf{M}$ be the one-turn matrix - i.e. the Jacobian of the one-turn map - and $\mathbf{z}$, an array of "regular" state coordinates in transverse phase space. Let $\mathbf{E}$ be a matrix whose columns are the eigenvectors of $\mathbf{M}$ correctly ordered and normalized. ${ }^{28}$ Then, the (complex) linear normal form coordinates defined over phase space are contained in the array a defined by the equation,

$$
\mathbf{a}=\mathbf{E}^{-1} \cdot \mathbf{z} .
$$

In particular - and this is the only case of interest in this memo - if linear motion is decoupled and we confine our attention to one plane only (the horizontal, here), then this becomes,

$$
a=\mathfrak{R} a+i \mathfrak{I} a=i \sqrt{I} e^{-i \varphi} \quad \text { and } \quad \sqrt{2 \beta} e^{-i(\psi-v \theta)} a=x+i\left(\alpha x+\beta x^{\prime}\right),
$$

where $\beta, \alpha$ and $\psi$ are the usual Courant-Snyder lattice functions. If $\left(x, x^{\prime}\right)$ are canonically conjugate, then so are $(\varphi, I)$ and $\left(a, a^{*}\right)$. The connections are

$$
d x \wedge d x^{\prime}=d \varphi \wedge d I=i d a \wedge d a^{*}=2 d(\Re a) \wedge d(\Im a) .
$$

Not surprisingly, the pair $(\varphi, I)$ are angle and action coordinates for the system. That last expression on the right implies that the area enclosed by a Jordan curve in $(\Re a, \mathfrak{I} a$ ) Cartesian space must be multiplied by two to be interpreted as "emittance." (This is nothing profound or mysterious. It is equivalent to writing that the area element in polar coordinates is $r d r d \theta=(1 / 2) d r^{2} d \theta$.) Finally, Hamilton's equation of motion is expressed in these coordinates as follows,

$$
i d a / d \theta=\partial H / \partial a^{*} .
$$

Nonlinear fields in the Hamiltonian: If we take $\theta=s / R$ to be the orbit parameter (aka "independent variable," "time"), then the Hamiltonians we shall use are written,

$$
H=v I=v a^{*} a-R A_{3} /|B \rho|,
$$

where $v$ is the (horizontal) tune, $R$ is the circumference of the closed orbit divided by $2 \pi, A_{3}$ is a local, nonlinear, longitudinal vector potential, expressing a transverse magnetic field, and $|B \rho|$ is the usual magnetic rigidity. In particular,

\footnotetext{
${ }^{27}$ If interested in further explanation, in abundant and nauseating detail, please read Reference [8].

${ }^{28}$ How that is done, along with a proof that it "always" can be done, can be found in Reference [25]. We shall not repeat those details here.
} 
in this memo we are interested only in quadrupoles, sextupoles and octupoles, so that,

$$
\begin{aligned}
\text { for a quadrupole : } A_{3} & =-\frac{B^{\prime}}{2 !}\left(x^{2}-y^{2}\right), \\
\text { for a sextupole : } A_{3} & =-\frac{B^{\prime \prime}}{3 !}\left(x^{3}-3 x y^{2}\right), \\
\text { and for an octupole : } A_{3} & =-\frac{B^{\prime \prime \prime}}{4 !}\left(x^{4}-6 x^{2} y^{2}+y^{4}\right) .
\end{aligned}
$$

The numerology of the principal resonances excited by multipoles is embedded within their exponents: for a quadrupole, $2 v_{x}$; for a sextupole, $3 v_{x}, v_{x}$ and $v_{x} \pm 2 v_{y}$; for an octupole, $4 v_{x}, 2 v_{x}, 2 v_{x} \pm 2 v_{y}, 4 v_{y}$ and $2 v_{y}$. Because we are considering horizontal motion only, by setting $y=0$ these fields simplify to $-B^{\prime} x^{2} / 2$ !, with principal resonance $2 v_{x},-B^{\prime \prime} x^{3} / 3$ !, with principal resonances $3 v_{x}$ and $v_{x}$, and $-B^{\prime \prime \prime} x^{4} / 4$ !, with principal resonances $4 v_{x}$ and $2 v_{x}$. We shall want to excite only the $3 v_{x}$ resonance, for third-integer extraction, and the $2 v_{x}$ resonances, for half-integer extraction.

First filter: shear and resonant terms: The procedure is to insert Eq.(29) into Eq.(33) and subsequently into Eq.(32) and apply perturbation theoretic manipulations to generate resonance exemplars. To begin with, sextupoles are used for to extract using the third-integer resonance. Thus, we consider

$$
\begin{aligned}
x^{3} & =(\beta / 2)^{3 / 2}\left(e^{-i(\psi-v \theta)} a+e^{i(\psi-v \theta)} a^{*}\right)^{3} \\
& =(\beta / 2)^{3 / 2}\left(e^{-i 3(\psi-v \theta)} a^{3}+e^{i 3(\psi-v \theta)} a^{* 3}+3 e^{-i(\psi-v \theta)} a^{2} a^{*}+3 e^{i(\psi-v \theta)} a a^{* 2}\right. \\
& =(\beta / 2)^{3 / 2}\left(e^{-i 3(\psi-v \theta)} a^{3}+e^{i 3(\psi-v \theta)} a^{* 3}+\cdots\right)
\end{aligned}
$$

The first two terms excite third-integer resonances; if we are not careful, the last two would excite integer resonances. We shall assume care and drop them. (Perturbatively, they still distort the phase space, an effect that will be ignored here. For a more thorough treatment, see [8].) The Hamiltonian is thus put into the form,

$$
H=v a^{*} a+\frac{1}{12 \sqrt{2}} \frac{B^{\prime \prime} R}{|B \rho|} \beta^{3 / 2}\left(e^{-i 3(\psi-v \theta)} a^{3}+e^{i 3(\psi-v \theta)} a^{* 3}\right)+\cdots
$$

These terms we retain; the rest we assume (here) can be "transformed away." 29

Next we consider half-integer extraction, controlled by octupoles and quadrupoles. Begin with the quadrupole component.

$$
\begin{aligned}
x^{2} & =(\beta / 2)\left(e^{-i(\psi-v \theta)} a+e^{i(\psi-v \theta)} a^{*}\right)^{2} \\
& =(\beta / 2)\left(2 a^{*} a+e^{-i 2(\psi-v \theta)} a^{2}+e^{i 2(\psi-v \theta)} a^{* 2}\right)
\end{aligned}
$$

If one is not careful, the first term will produce an overall tune shift (compare to Eq.(32)). Again, we assume care will be taken not to let that happen and drop that term. (Or, think of it as being "renormalized" away by absorption into the definition of $v$.) The second and third terms distort orbits and, more to the point, can excite half-integer resonances. Octupoles will contribute as follows.

$$
\begin{aligned}
x^{4} & =(\beta / 2)^{2}\left(e^{-i(\psi-v \theta)} a+e^{i(\psi-v \theta)} a^{*}\right)^{4} \\
& =(\beta / 2)^{2}\left(6\left(a^{*} a\right)^{2}+4 e^{-i 2(\psi-v \theta)} a^{3} a^{*}+4 e^{i 2(\psi-v \theta)} a a^{* 3}+e^{-i 4(\psi-v \theta)} a^{4}+e^{i 4(\psi-v \theta)} a^{* 4}\right)
\end{aligned}
$$

\footnotetext{
${ }^{29}$ This is not merely "hand-waving," but making this precise would fill several more pages. Suffice it to say that, within the phase space region of interest, the sextupoles are distributed so as to produce no "significant" resonance excitations at higher orders in perturbation theory. The phase space will be distorted but no further separatrices appear, at the scale of interest. Please see Reference [8] for further details.
} 
The first term has no dependence on phases and produces "shear," i.e. amplitude dependence of the tune. The second and third excite half-integer resonances, while the fourth and fifth excite fourth-integer resonances. In the configuration of magnets assumed in this memo, we assert that care has been taken to avoid both. That is, we shall drive the half-integer resonance with quadrupoles only and use the octupoles only to move the tune onto the half-integer as the orbit amplitude increases. Accordingly, we take

$$
x^{4}=(\beta / 2)^{2}\left(6\left(a^{*} a\right)^{2}+\cdots\right),
$$

which puts the Hamiltonian into the form,

$$
H=v a^{*} a+\frac{1}{16} \frac{B^{\prime \prime \prime} R}{|B \rho|} \beta^{2}\left(a^{*} a\right)^{2}+\frac{1}{4} \frac{B^{\prime} R}{|B \rho|} \beta\left(e^{-i 2(\psi-v \theta)} a^{2}+e^{i 2(\psi-v \theta)} a^{* 2}\right)+\cdots
$$

Second filter: averaging: The rationale for the next step, heuristically called "averaging," is embedded in perturbation theory. It is frequently motivated intuitively, but it is justified fully only within the context of a rigorous perturbation theory. As a result, the coefficient of $a^{m}$ is replaced with a single harmonic term, $e^{-i n \theta}$, where $n$ minimizes the value of $|m v+n|$. In particular, in the Hamiltonian of Eq.(35), we shall replace

$$
\frac{1}{12 \sqrt{2}} \frac{B^{\prime \prime} R}{|B \rho|} \beta^{3 / 2} e^{-i 3(\psi-v \theta)} \approx G_{3} e^{-i n \theta}
$$

where $3 v+n \approx 0$. (Specifically, for the debuncher, $n=-29$.) This brings us to the Hamiltonian form,

$$
H=v \cdot a^{*} a+G_{3} e^{-i n \theta} a^{3}+G_{3}^{*} e^{i n \theta} a^{* 3}+\cdots .
$$

Correspondingly, for the half-integer resonance, in Eq.(B) we replace

$$
\begin{aligned}
\frac{1}{16} \frac{B^{\prime \prime \prime} R}{|B \rho|} \beta^{2} & \approx G_{4}+\cdots \\
\frac{1}{4} \frac{B^{\prime} R}{|B \rho|} \beta e^{-i 2(\psi-v \theta)} & \approx G_{2} e^{-i n \theta}+\cdots,
\end{aligned}
$$

which gives us the following.

$$
H=\Delta \cdot a^{*} a+G_{4}\left(a^{*} a\right)^{2}+G_{2} e^{-i n \theta} a^{2}+G_{2}^{*} e^{i n \theta} a^{* 2}+\cdots
$$

Autonomous exemplars: The final incantation - and perhaps the most valid - is intoned in order to produce autonomous exemplars. Equations (40) and (39) still depend explicitly on $\theta$ through its appearance in the harmonic factors. Rewriting Eq.(40) as

$$
H=v \cdot a^{*} a+G_{3}\left(a e^{-i n \theta / 3}\right)^{3}+G_{3}^{*}\left(a e^{-i n \theta / 3}\right)^{* 3}+\cdots
$$

makes obvious what the required transformation should be.

$$
a e^{-i n \theta / 3} \mapsto a
$$

A bit of nomenclature: Forest and Irwin [26] descriptively called this a transformation to a "co-moving frame." In principle, we should change the symbol used for the coordinate but will not. Please consider that at this point the symbol 
has been "overloaded" and henceforth refers to the co-moving frame. Simultaneously, to retain Hamilton's equation of motion, Eq.(31), we must add a term to the Hamiltonian. ${ }^{30}$ The final exemplar looks as follows:

$$
H=\Delta \cdot a^{*} a+G_{3} a^{3}+G_{3}^{*} a^{* 3}+\cdots,
$$

where $\Delta=\mathrm{v}+n / 3 \approx 0$. It has the form appropriate to an autonomous dynamical system, making the Hamiltonian a constant of the motion, that being the entire point of this final transformation.

Similarly, for the half-integer we rewrite Eq.(39) as,

$$
H=v \cdot a^{*} a+G_{4}\left(a^{*} a\right)^{2}+G_{2}\left(a e^{-i n \theta / 2}\right)^{2}+G_{2}^{*}\left(a e^{-i n \theta / 2}\right)^{* 2}+\cdots
$$

Then, the transformation,

$$
a e^{-i n \theta / 2} \mapsto a
$$

gives us,

$$
H=\Delta \cdot a^{*} a+G_{4}\left(a^{*} a\right)^{2}+G_{2} a^{2}+G_{2}^{*} a^{* 2}+\cdots,
$$

with $\Delta=\mathrm{v}+n / 2 \approx 0$.

Neglected principal terms: Terms that were dropped in going from the expansions in Equations (34), (36) and (37) to the exemplars in Equations (43) and (41) can excite additional principal resonances: half-integer and quarter-integer, for the quadrupoles and octupoles; an integer resonance, for the sextupoles. There is little to be concerned about in the case of the sextupoles, as a tune near a third-integer is nowhere near an integer. Not so, with the octupoles; a tune near a half-integer is also unavoidably near a quarter-integer: that is, $2 v_{x} \approx 19 \Rightarrow 4 v_{x} \approx 38$. Neglecting these resonances cannot be justified unless care is taken to avoid exciting them when distributing the control magnets. In particular, as only the zeroth harmonic octupole term is wanted for the exemplar, octupoles must be arranged so that the second and fourth harmonics of their distribution vanish. As only the second harmonic coefficient is wanted from the quadrupole circuit, they are arranged so as not to excite the zeroth harmonic; i.e. the harmonic quad circuit should not affect the tune. These requirements are most readily accomplished by exploiting the ring's symmetry. When the symmetry is broken, either deliberately or from random shifts in phase advance induced by quadrupole field errors, compensation must be made by tuning to reestablish a "base point" for control settings. Figure 22 illustrates just one class of separatrices to arise - and, in our case, the most likely one - if this is not done. ${ }^{31}$ If necessary, fine tuning could be carried out on individual elements. Even so, it would be a challenge to restore the separatrix completely to its symmetric form.

\footnotetext{
${ }^{30}$ There is nothing mysterious about this; we need not invoke anything as complicated as generating functions. It simply follows from the fundamental rule for derivatives: $(c a)^{\prime}=c^{\prime} a+c a^{\prime}$.

${ }^{31}$ It could be argued that distortion is more dangerous than the additional branches of the separatrix, as they are totally contained within the lobes of the other branches.
} 


\section{Linearly independent control circuits}

Each of the complex coupling parameters $G_{2}$, and $G_{3}$, written in Equations (2) and (15) for third-integer and half-integer extraction respectively, requires two control circuits to set its amplitude and phase (or real and imaginary parts). Ideally these would be orthogonal - that is, their contributions would be orthogonal in the complex plane - but all that is necessary is that they be linearly independent.

Suppose that the desired complex coupling constant is $g$. (I.e. this is the resonance sum.) We have two control circuits, currently producing $g_{1}$ and $g_{2}$ respectively and seek the superposition,

$$
g=s_{1} g_{1}+s_{2} g_{2}
$$

where $s_{1}$ and $s_{2}$ are real. This is solved in identical fashion to the same problem in real vector spaces.

$$
\begin{aligned}
i g_{2}^{*} g & =i s_{1} g_{2}^{*} g_{1}+i s_{2}\left|g_{2}\right|^{2} \\
\mathfrak{R}\left(i g_{2}^{*} g\right) & =s_{1} \mathfrak{R}\left(i g_{2}^{*} g_{1}\right) \\
\text { Thus, } s_{1} & =\mathfrak{I}\left(g_{2}^{*} g\right) / \mathfrak{I}\left(g_{2}^{*} g_{1}\right) \\
\text { and similarly, } s_{2} & =\mathfrak{I}\left(g_{1}^{*} g\right) / \mathfrak{I}\left(g_{1}^{*} g_{2}\right) .
\end{aligned}
$$

Though unnecessary, this could be writtin in polar form. Let $g=|g| e^{i \phi}, g_{1}=\left|g_{1}\right| e^{i \phi_{1}}$, and $g_{2}=\left|g_{2}\right| e^{i \phi_{2}}$. Then,

$$
\begin{aligned}
& s_{1}=\frac{|g|}{\left|g_{1}\right|} \frac{\sin \left(\phi-\phi_{2}\right)}{\sin \left(\phi_{1}-\phi_{2}\right)} \\
& s_{2}=\frac{|g|}{\left|g_{2}\right|} \frac{\sin \left(\phi-\phi_{1}\right)}{\sin \left(\phi_{2}-\phi_{1}\right)} .
\end{aligned}
$$

Of course, since the coupling constants are linear functionals of magnetic fields, it is always possible to apply singular value decomposition (SVD) to find truly orthogonal combinations of elements strengths. Figure 23 illustrates one example that was carried out on the locations of focusing quadrupoles in the straight sections. From it can be deduced the relative strengths that can be assigned to octupoles placed at those locations in order to supply the zeroth harmonic for half-integer extraction without exciting the second and fourth harmonics. These are the lines colored black. At the same time, two red lines indicate how to excite orthogonal contributions to the second harmonic without exciting the fourth. We shall not now explain this method further but only note in passing that, in principle, it is "always" possible to use SVD to find orthogonal circuits, though, in operational practice, it will be better to rely on symmetries and phase advances specifically designed to create useful phasors. 


\section{Debuncher design lattice file}

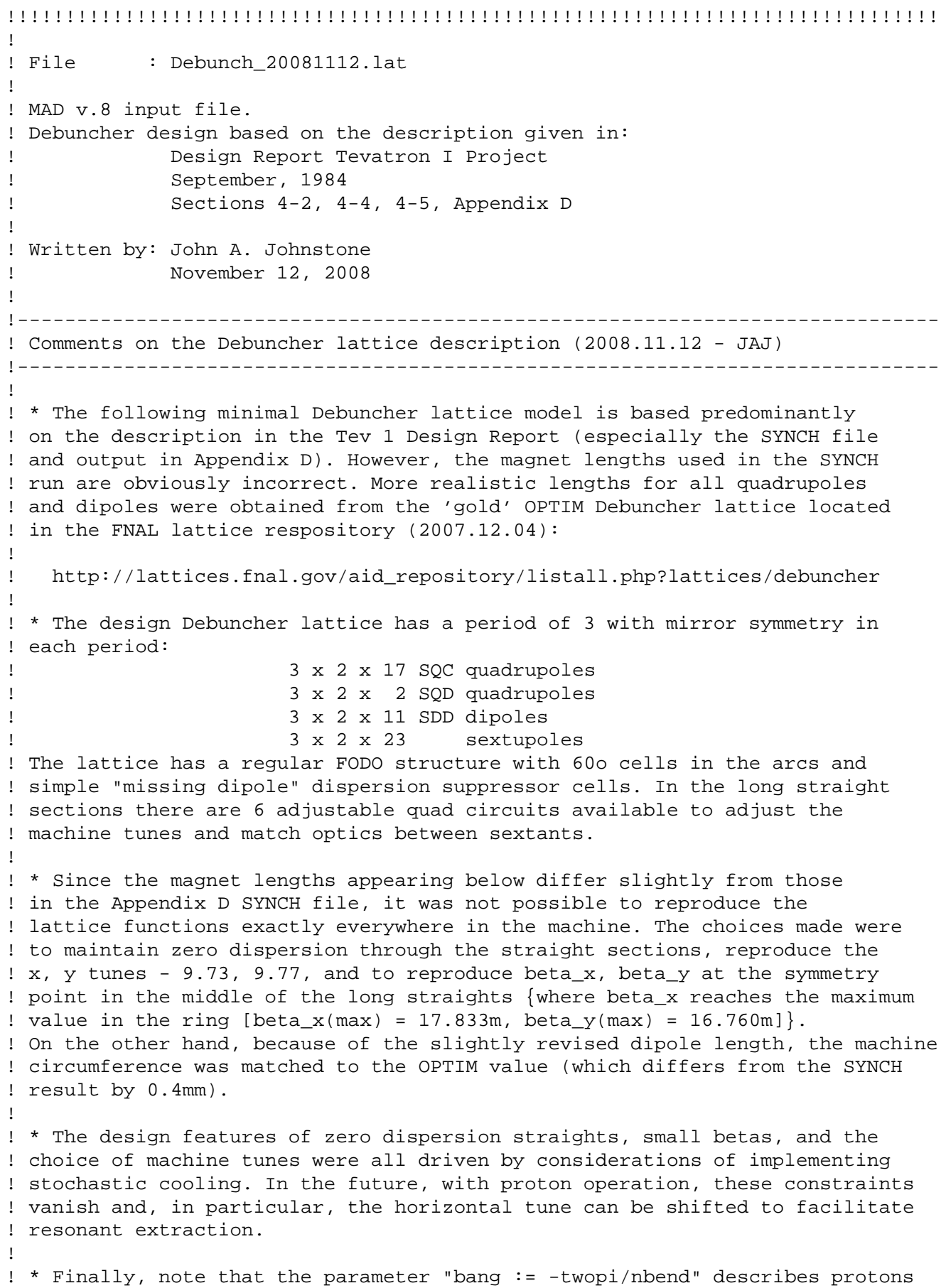




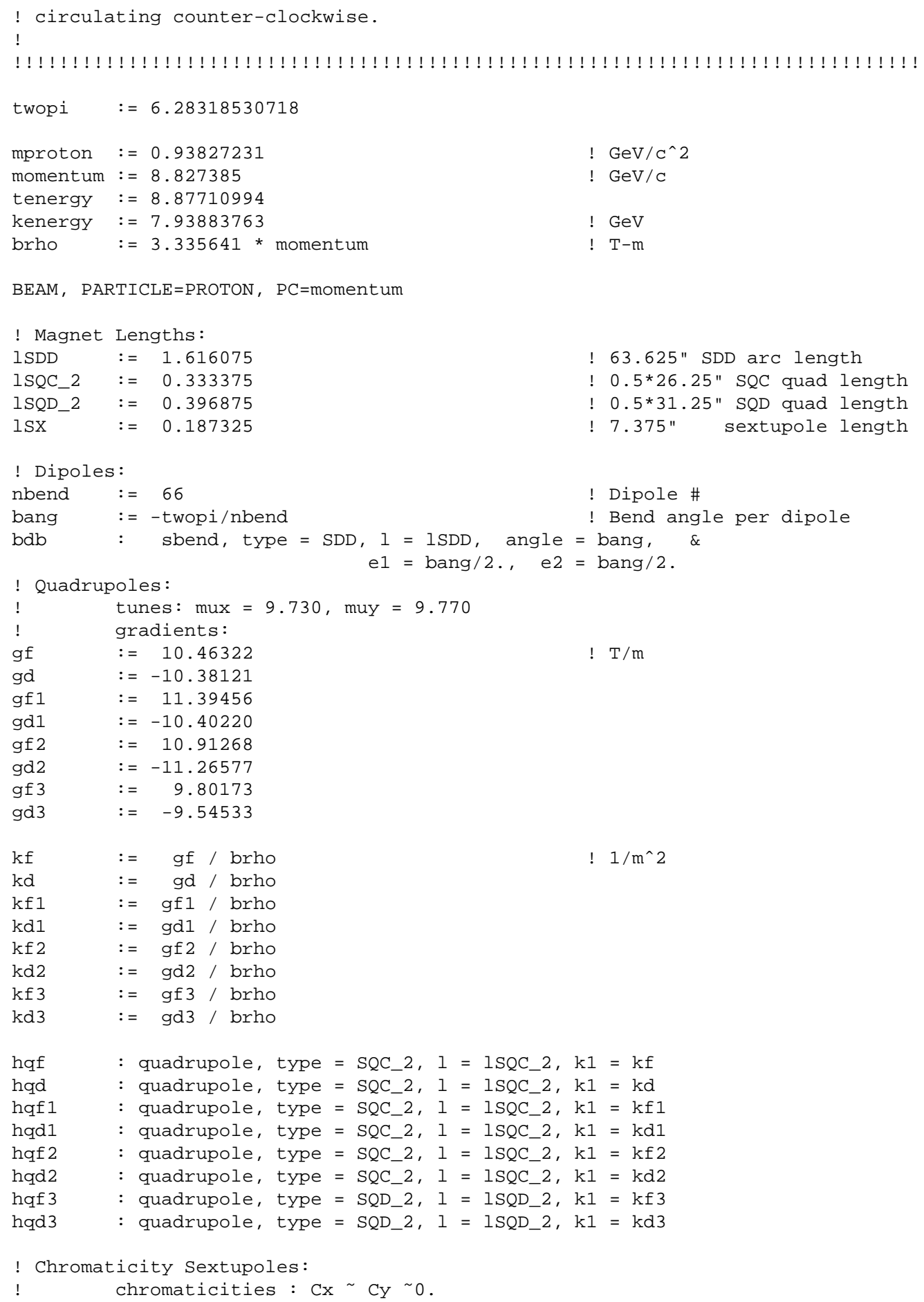




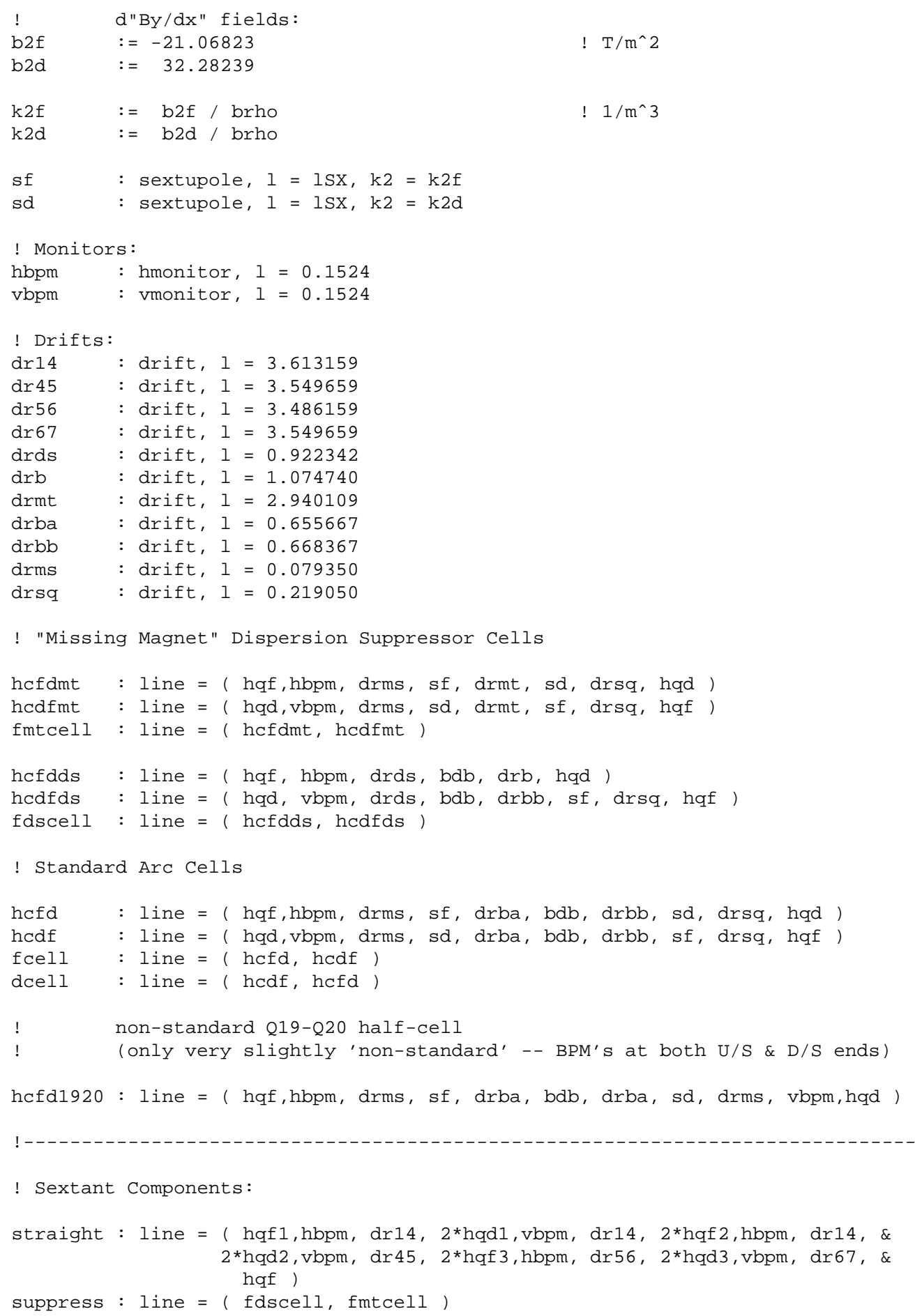




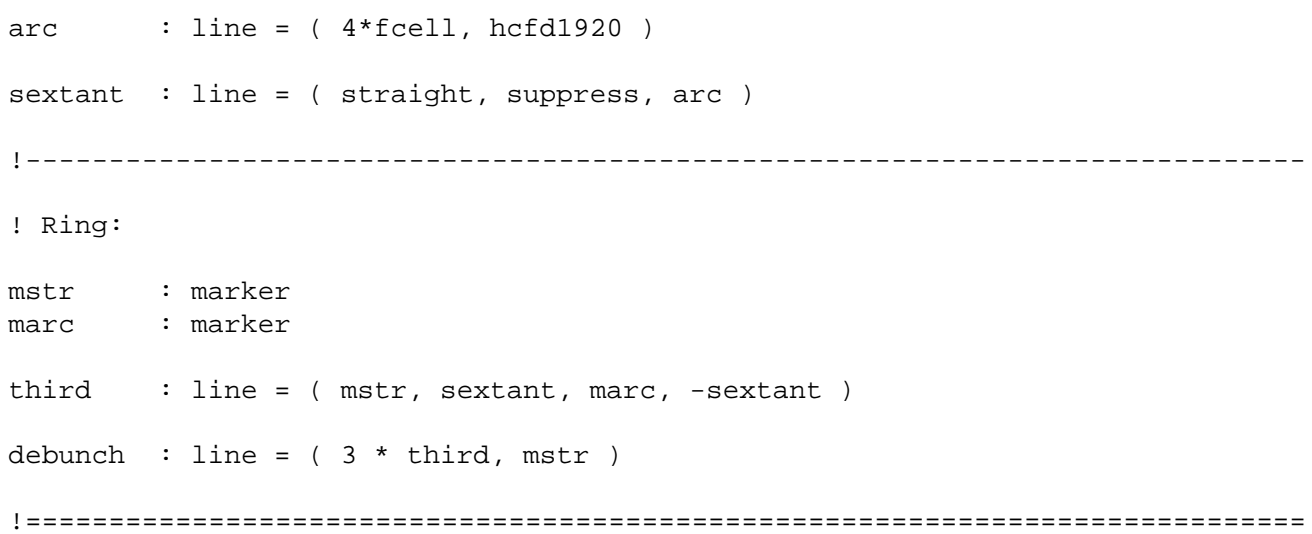




\section{E Accumulator design lattice file}

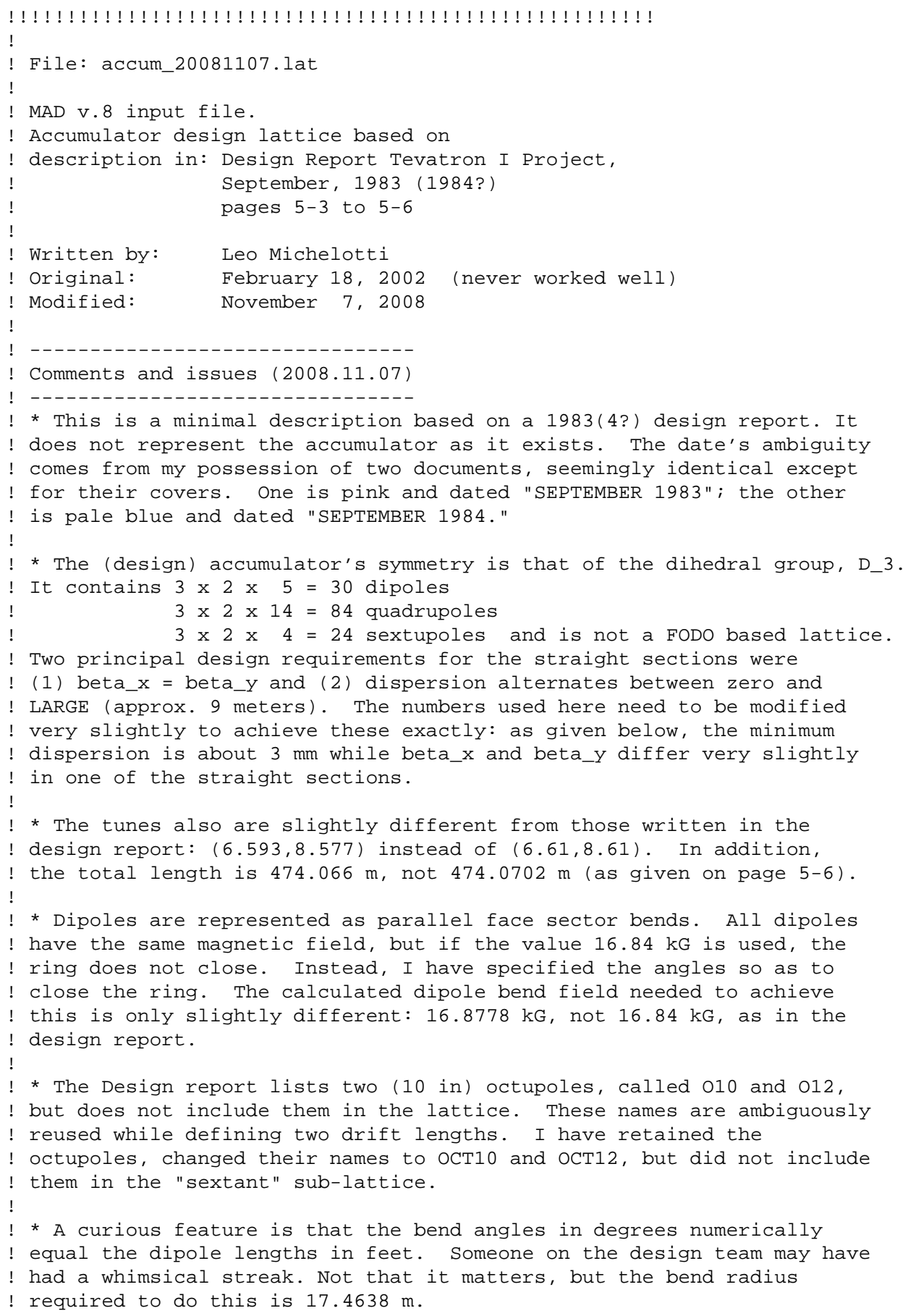




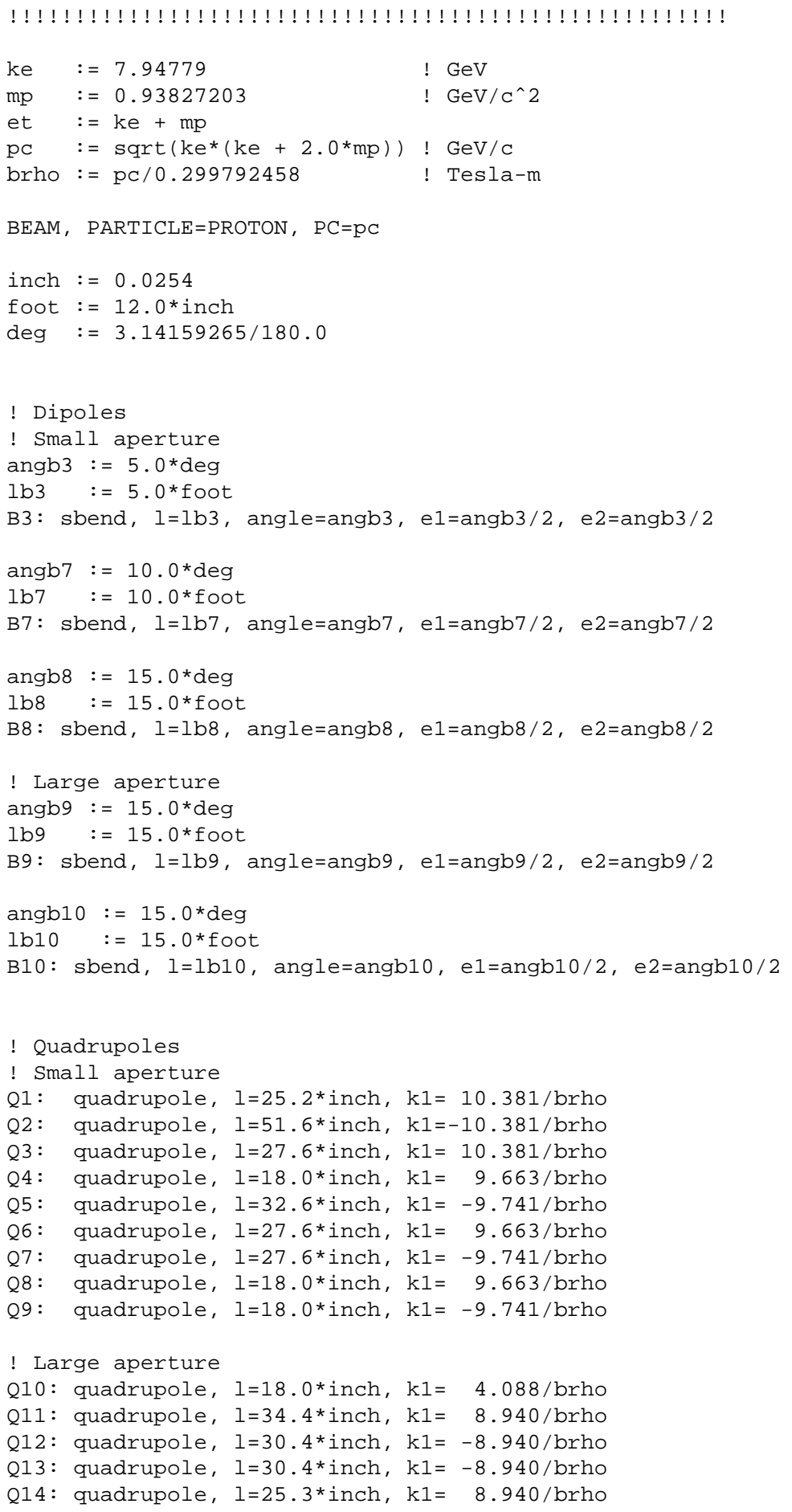




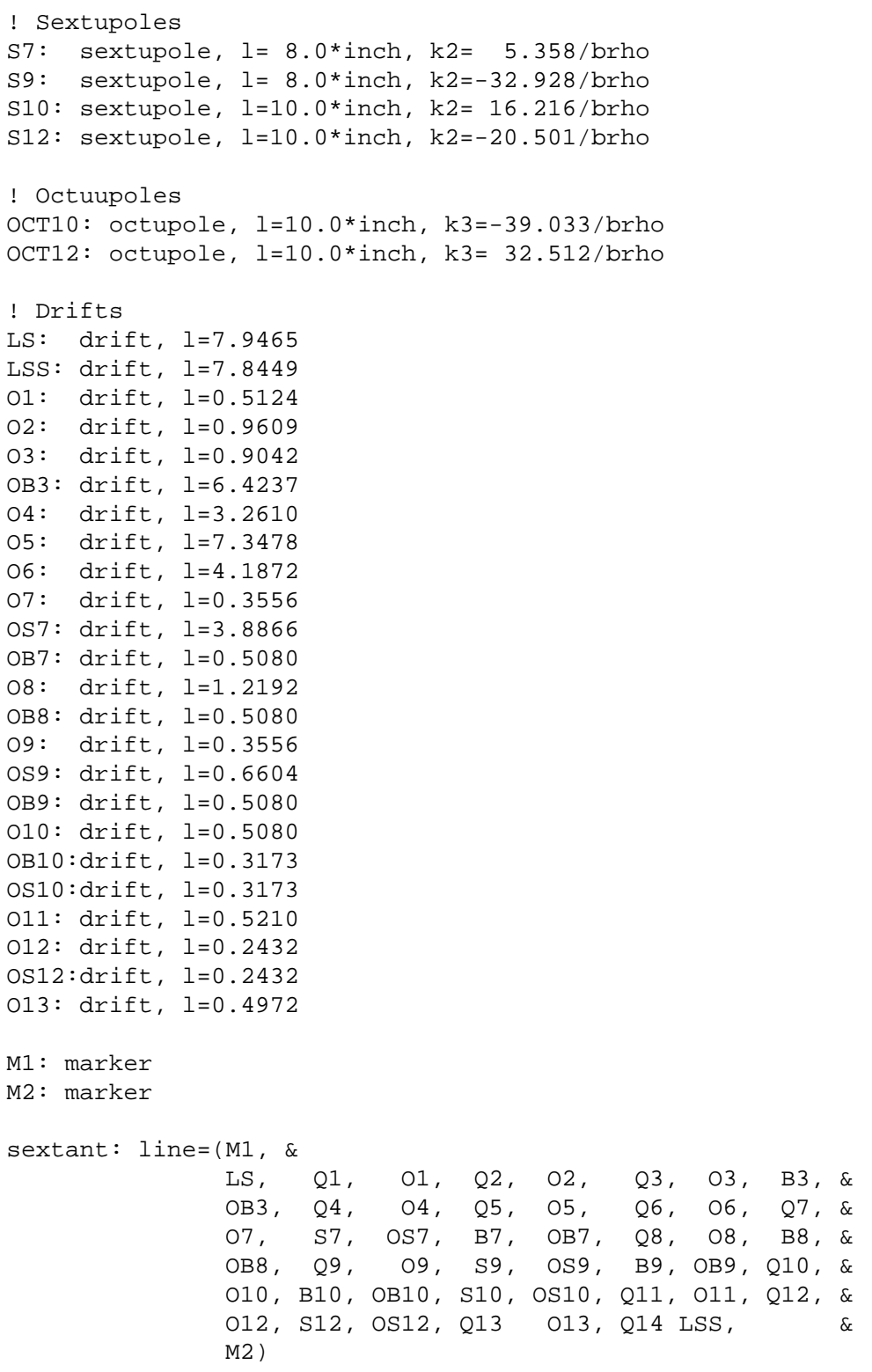




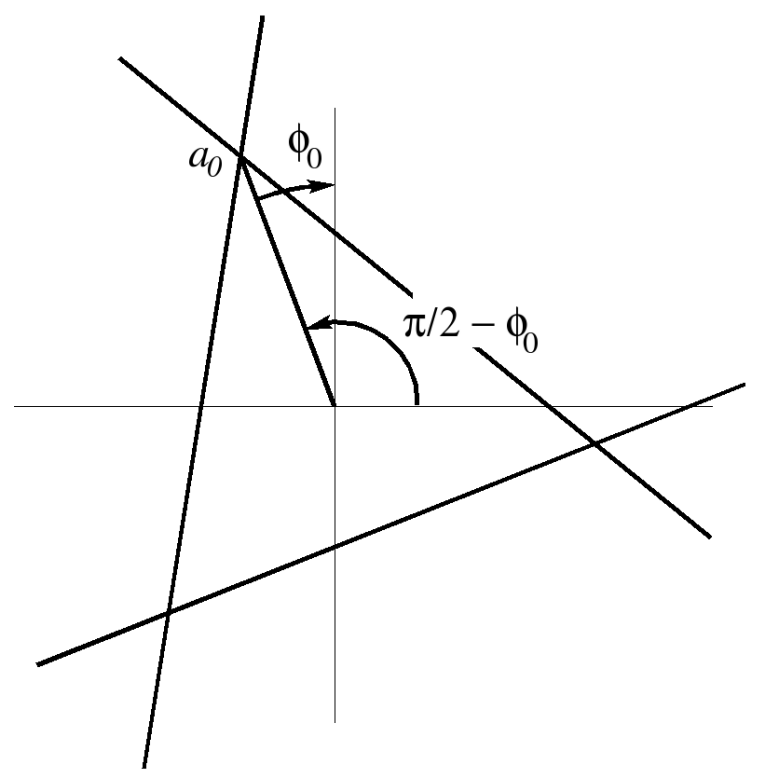

Figure 1: Angle connecting a "standard" triangle with the separatrix is $\pi / 2-\varphi_{0}, \operatorname{not} \varphi_{0}$.

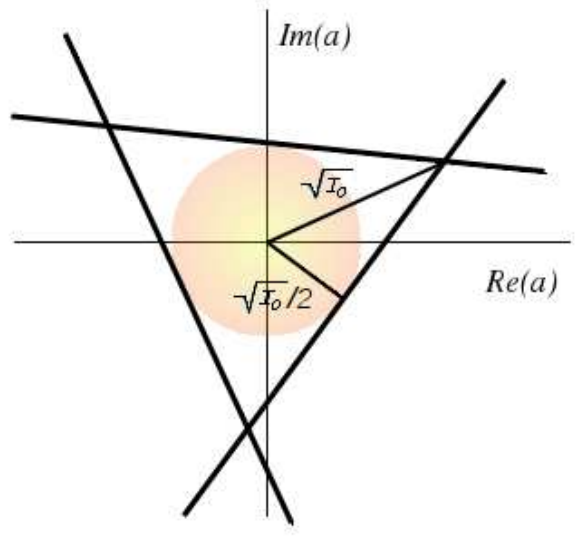

Figure 2: Central emittance of the third-integer resonance exemplar. Using these coordinates, "areas" are multiplied by 2 to obtain emittances. Alternatively, the length scale is multiplied by $\sqrt{2}$. (See Eq.(30) in Appendix B.) 


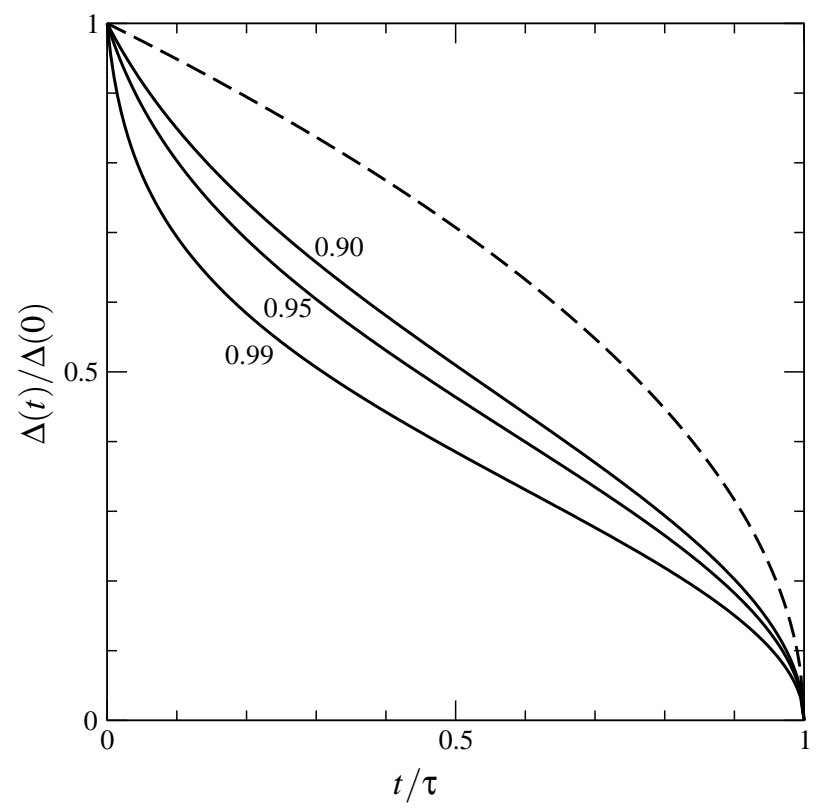

Figure 3: Suggested ramp profiles for constant spill rate. The dashed curve at the top was calculated using an assumption of constant density; the three bottom curves assume truncated Gaussians where 90\%-99\% of the initial emittance is enclosed at the start.
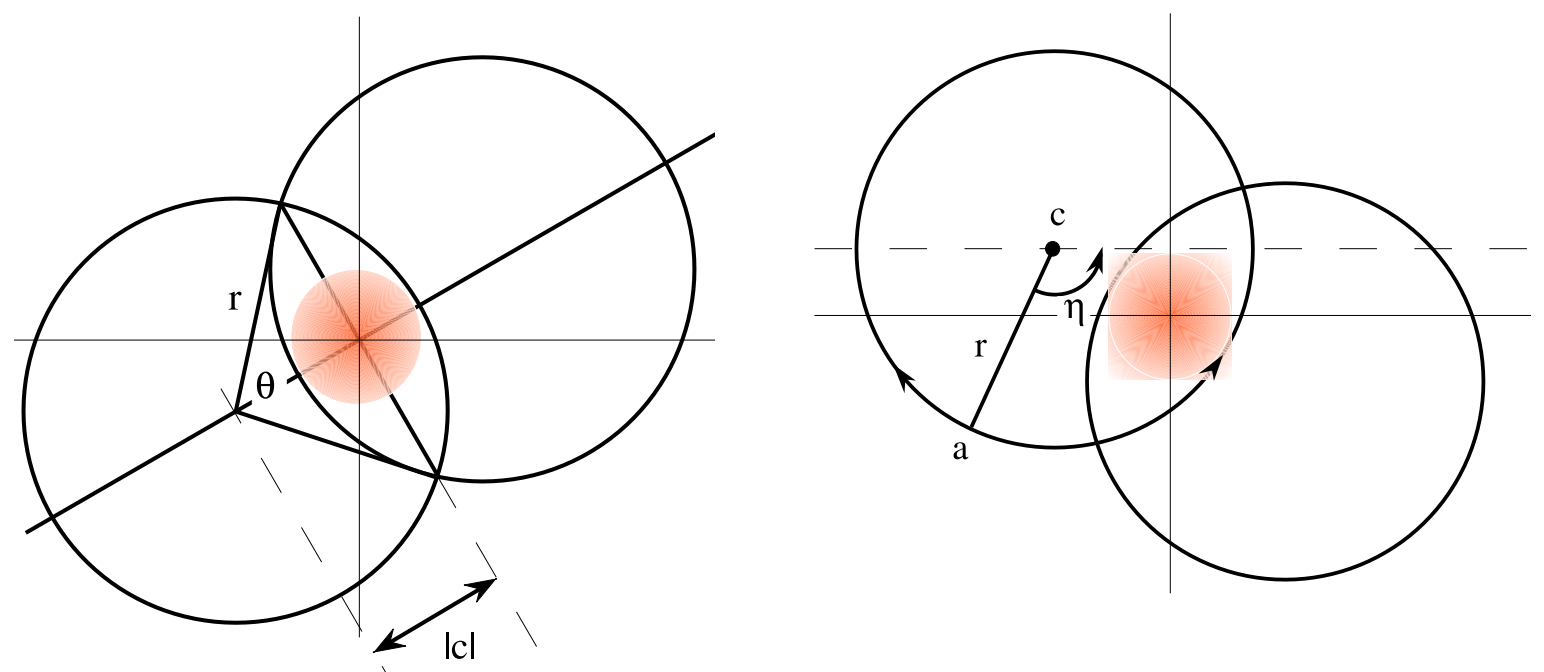

Figure 4: Central emittance of the half-integer resonance exemplar. The colored circle indicates a bunch at the onset of extraction. On the right, arrows on the separatrix show the direction of the (faux) "flow." The separatrices are flipped to confuse the reader. 


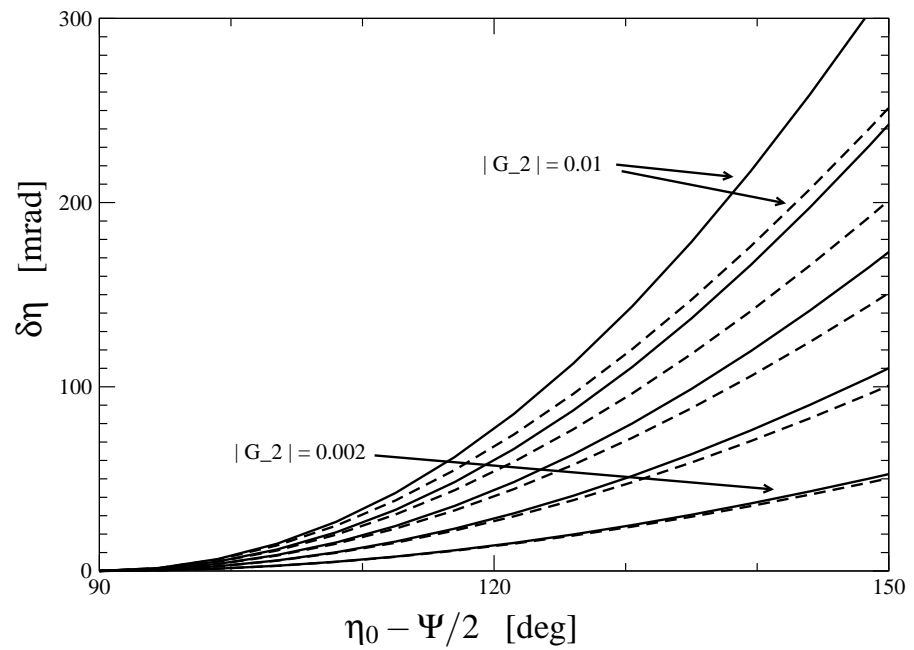

Figure 5: Comparison of exact step size with approximation, for the half-integer resonance exemplar with tangential lobes, and for $\left|G_{2}\right| \in\{0.002,0.004,0.006,0.008,0.01\}$.

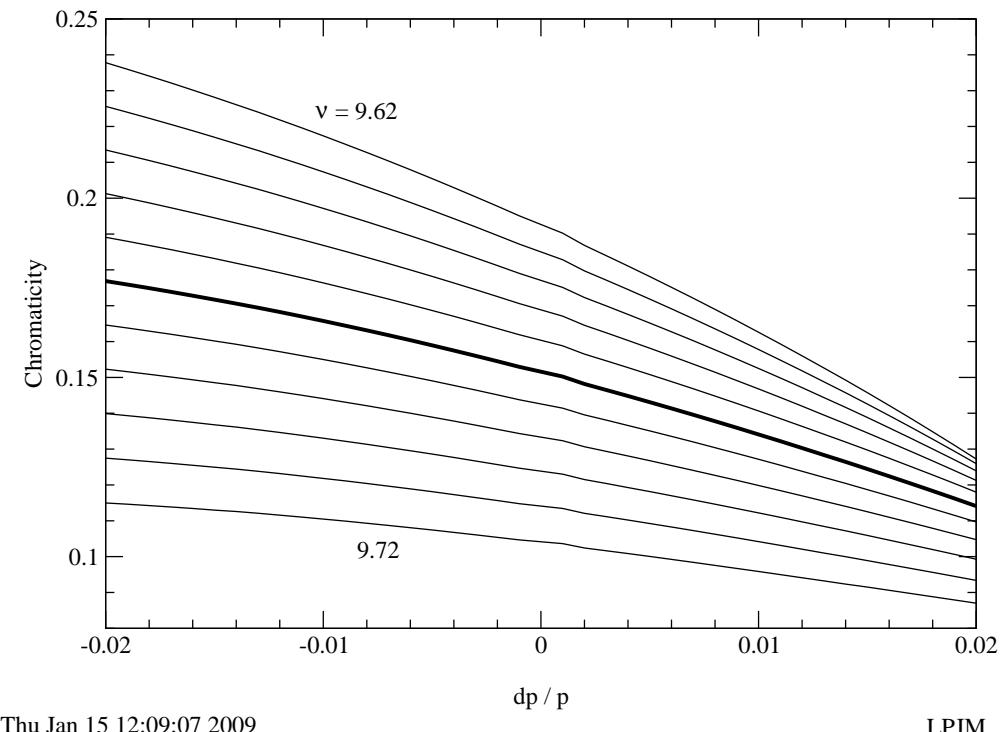

Figure 6: This was the result of a chromaticity scan carried out on the Debuncher model, whose chromaticity had been set explicitly to (near) zero. 


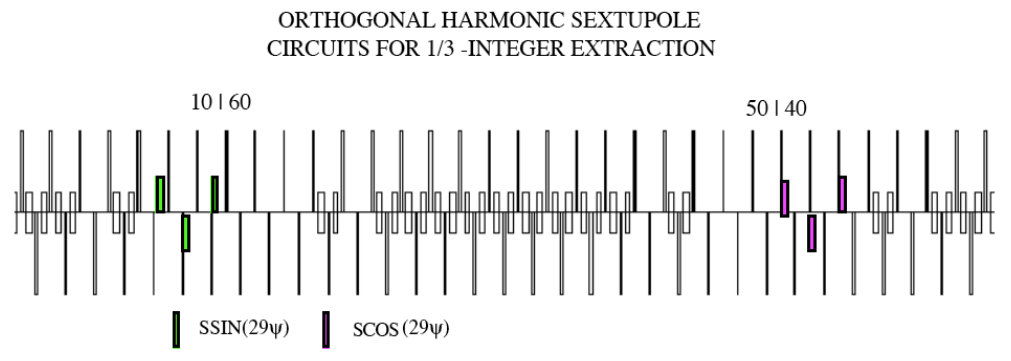

Figure 7: Location of harmonic sextupoles on two orthogonal circuits configured for third-integer resonance extraction.

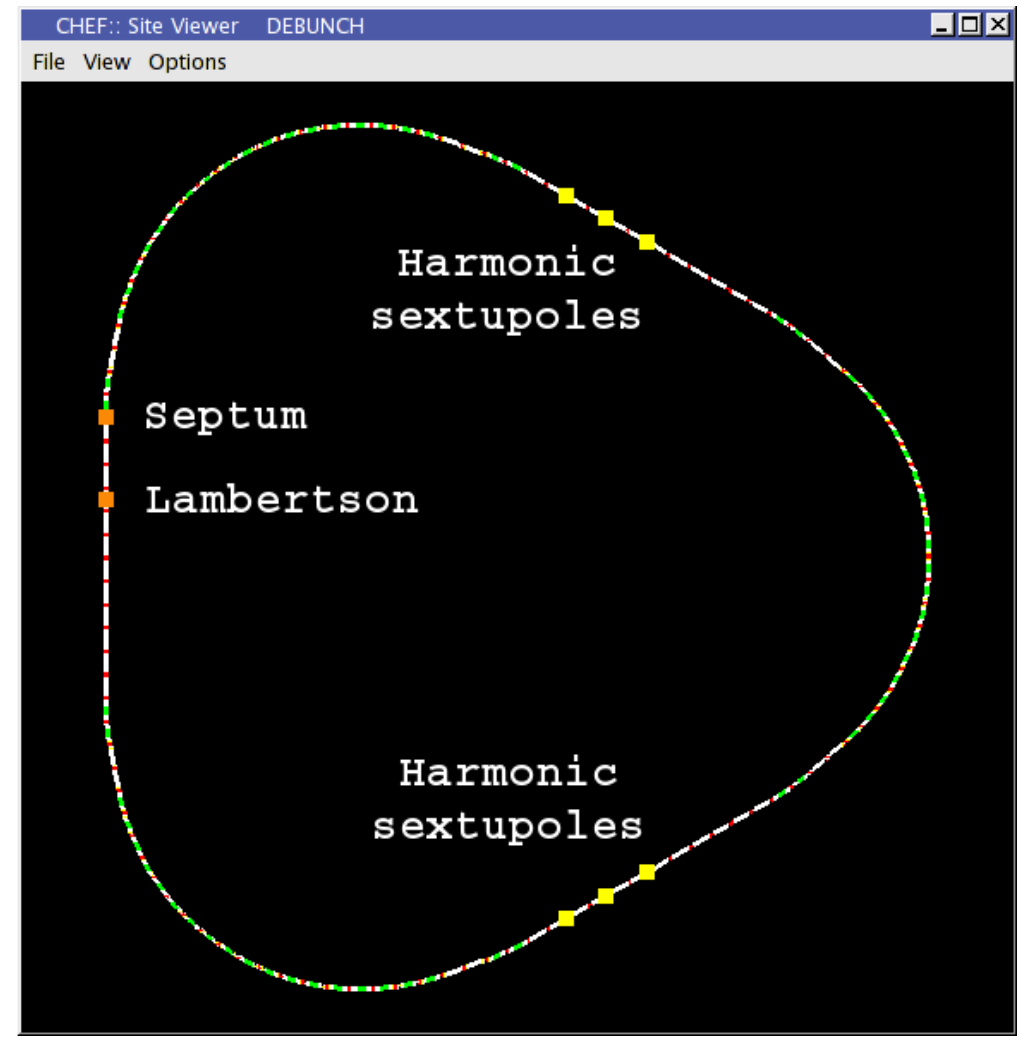

Figure 8: Locations of the harmonic sextupoles, septum and lambertson for the third-integer resonance model. The color scheme is: quadrupoles, red; dipoles, green; sextupoles (including chromaticity sextupoles), yellow; septum and lambertson, orange. 

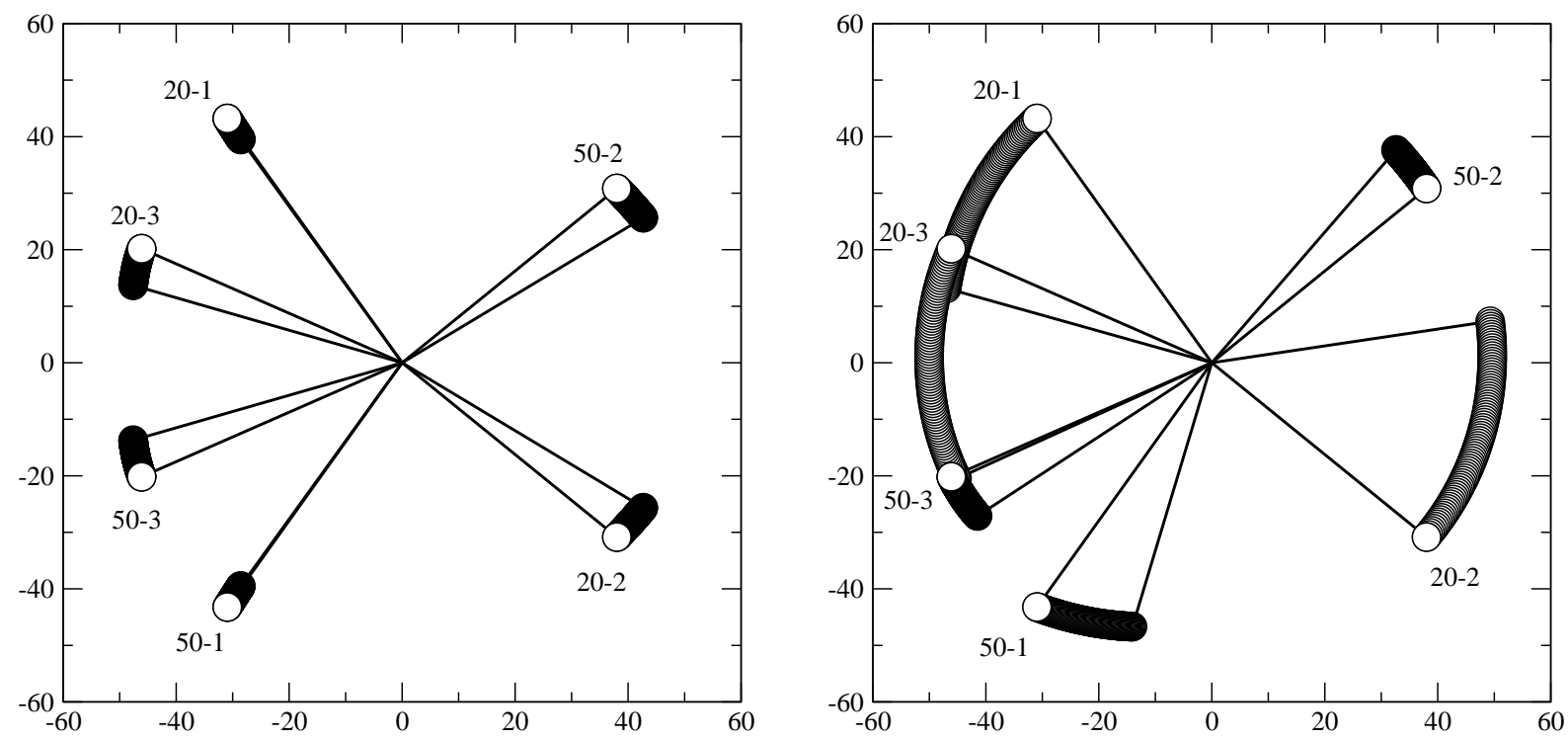

Figure 9: Correct (left) and incorrect (right) phasors for the third-integer resonance during the squeeze

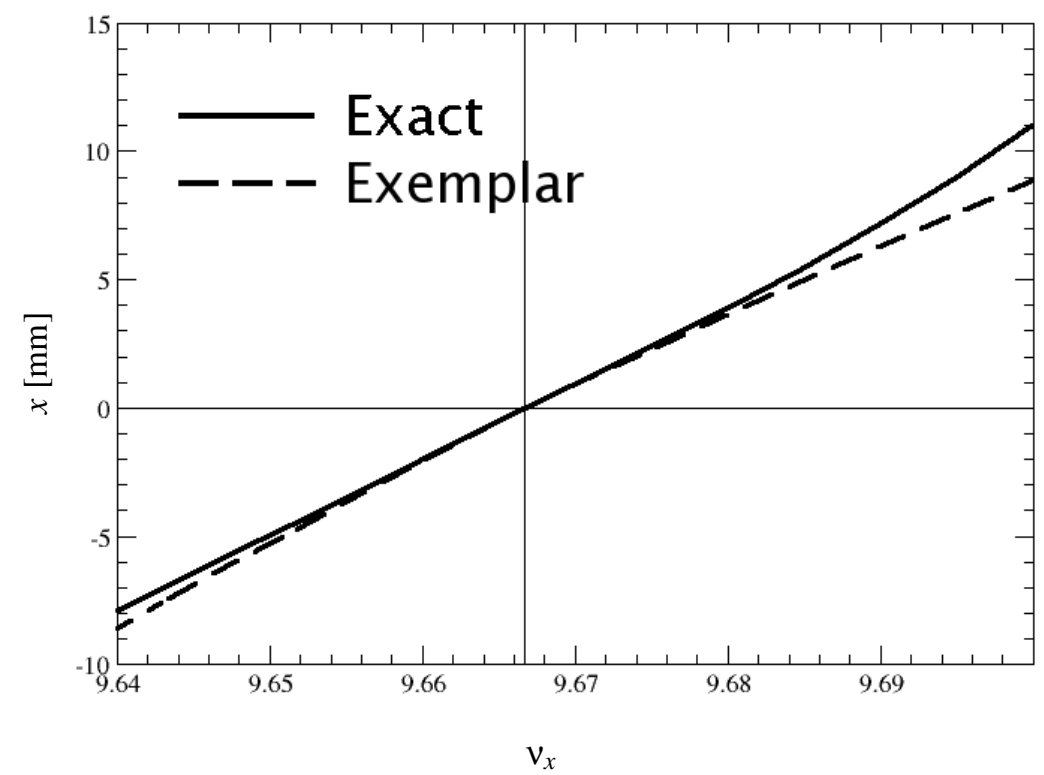

Figure 10: Comparison between exact resonant orbit and as predicted by exemplar. 


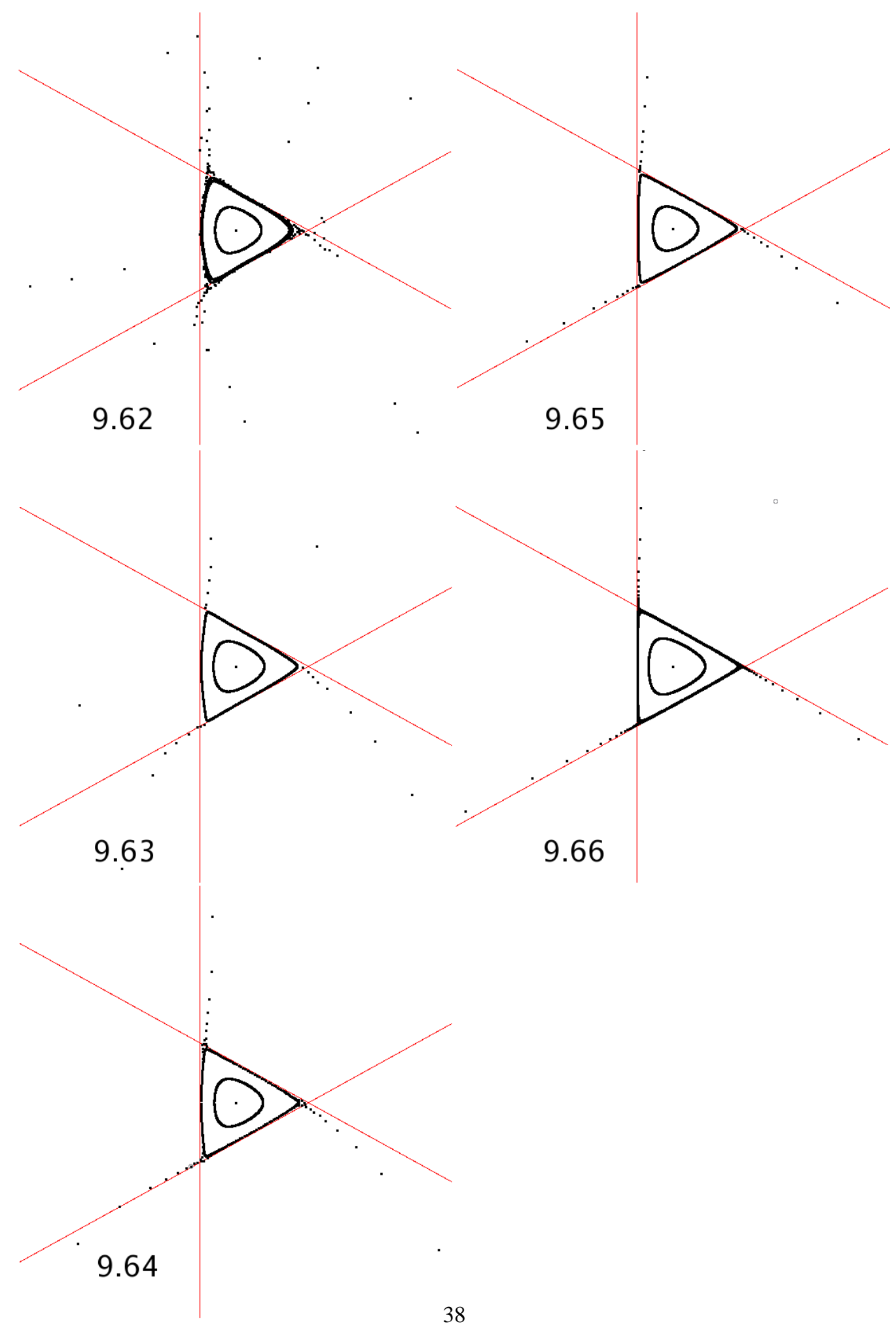

Figure 11: Comparison of exact separatrix, from tracking, to the exemplar, for $v=9.62,9.63, \ldots, 9.66$. The invariant emittance of the central stable region is $\beta \gamma \varepsilon / \pi=20 \mathrm{~mm}-\mathrm{mr}$ in each figure. To accomplish this, the magnitude of each sextupole's integrated strength changed linearly with tune from $\approx 15 \mathrm{~T} / \mathrm{m}$ at $v_{x}=9.66$ to $\approx 107 \mathrm{~T} / \mathrm{m}$ at $v_{x}=9.62$. 


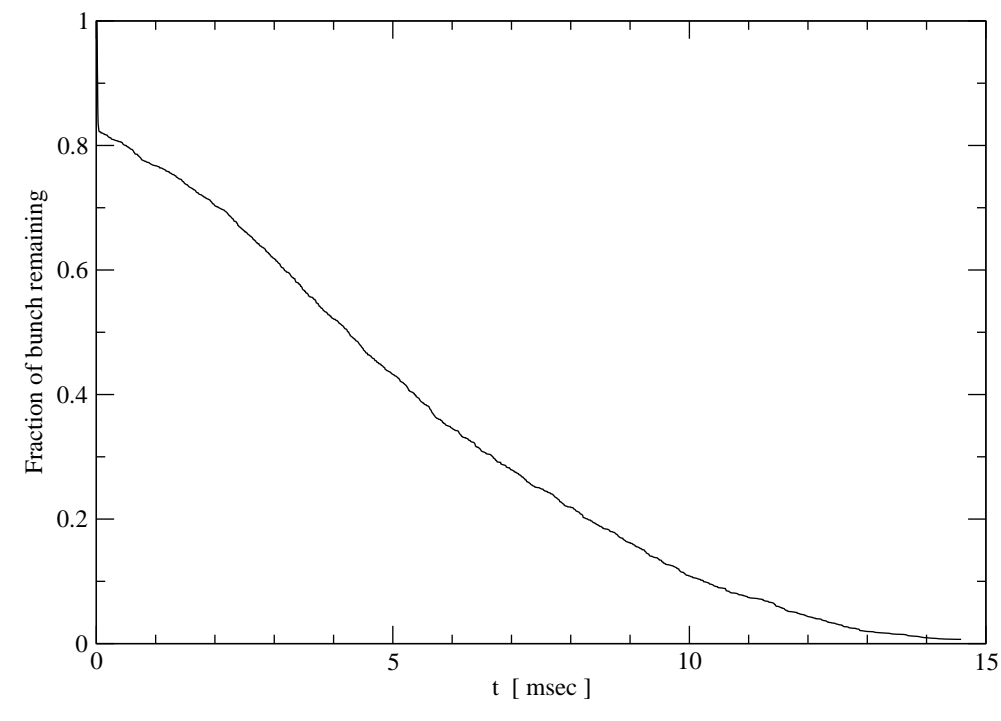

Fri Jan 30 13:34:25 2009

LPJM

Figure 12: History of the spill during a simulated extraction from the Debuncher using the third-integer resonance. The ramp was taken to be linear throughout the squeeze; no care was taken to control the spill. (For this run, the initial bunch contained 2048 protons.) Over several simulations with different initial distributions, initial rapid loss removed 10-20\% of the bunch.

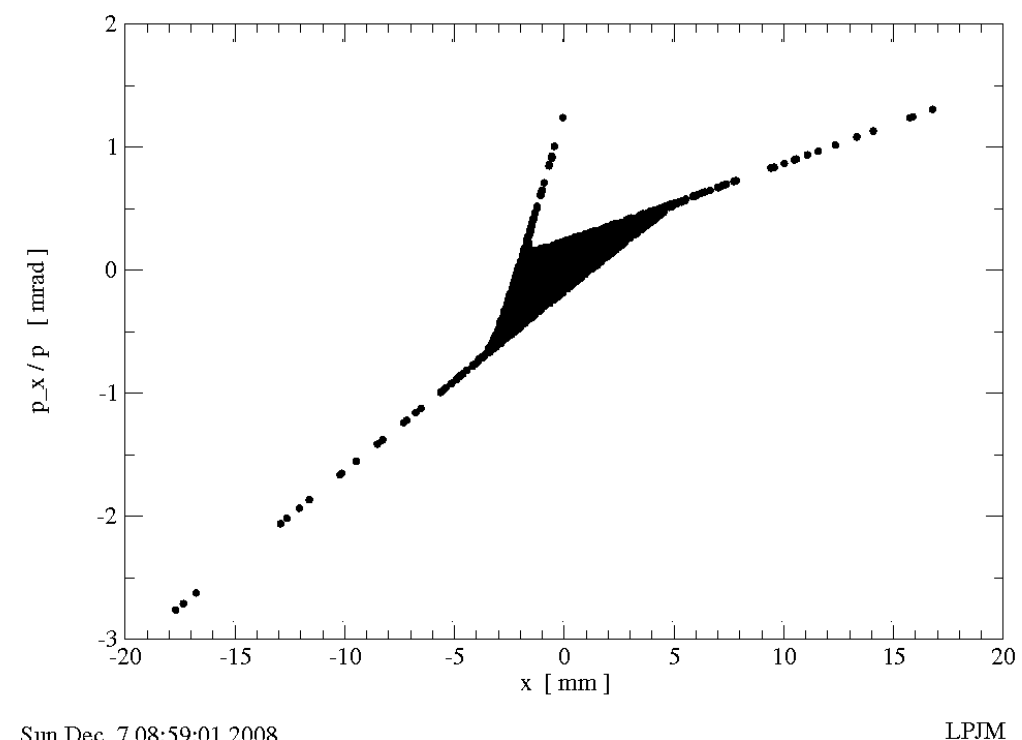

Figure 13: Scatter diagram of states in horizontal phase space (more or less) midway through a "simple" simulated extraction using the third-integer resonance. "Simple" means only horizontal coordinates were non-zero when populating the initial bunch. In some sense, then, this represents a "best case scenario." 
$1 / 2$ integer resonance phasors

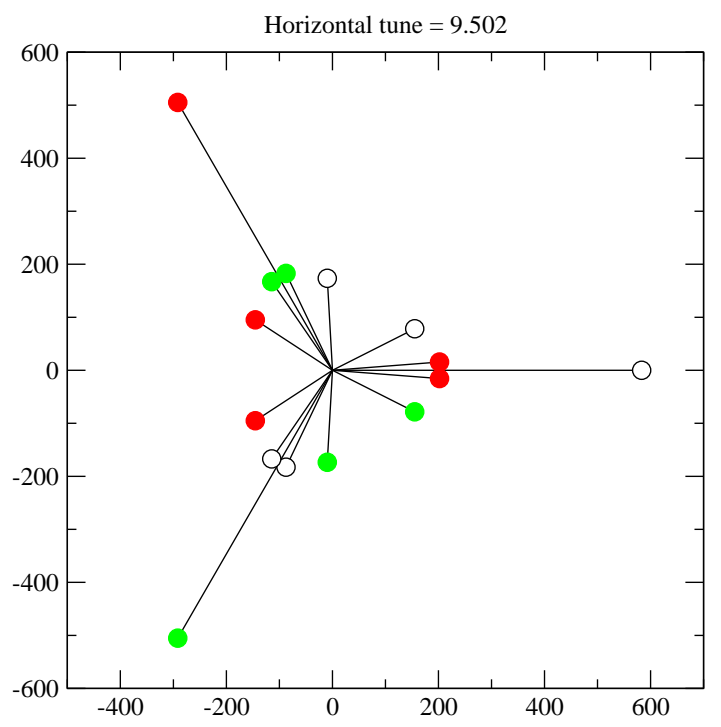

Thu Mar 5 09:52:53 2009

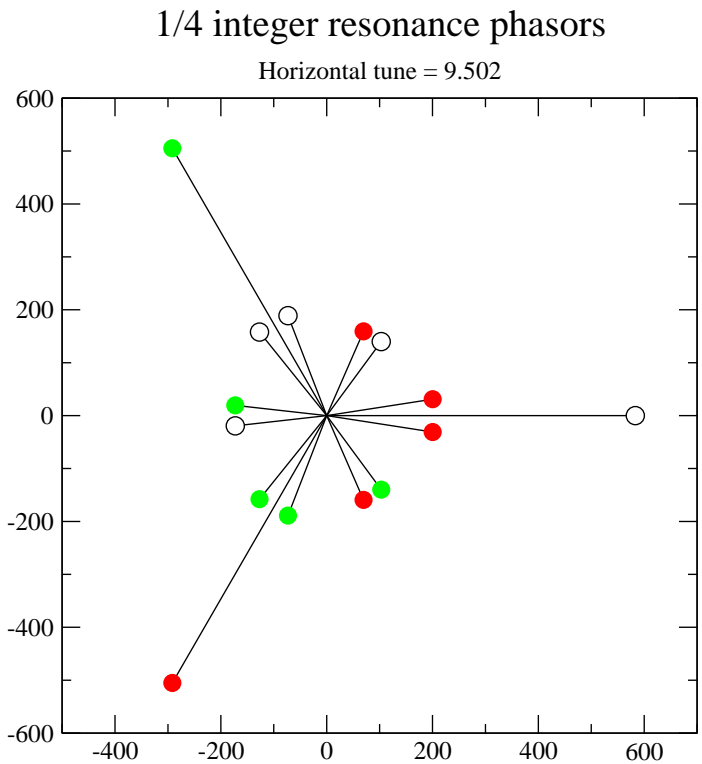

Thu Mar 5 11:37:07 2009

LPJM

Figure 14: Phasor diagrams for the $2\left(\psi_{x}-v_{x} \theta\right)$ and the $4\left(\psi_{x}-v_{x} \theta\right)$ terms in the resonance functionals (sums, integrals, whatever), for the minimalist configuration. Colors identify contributions from the three sectors in the Debuncher.

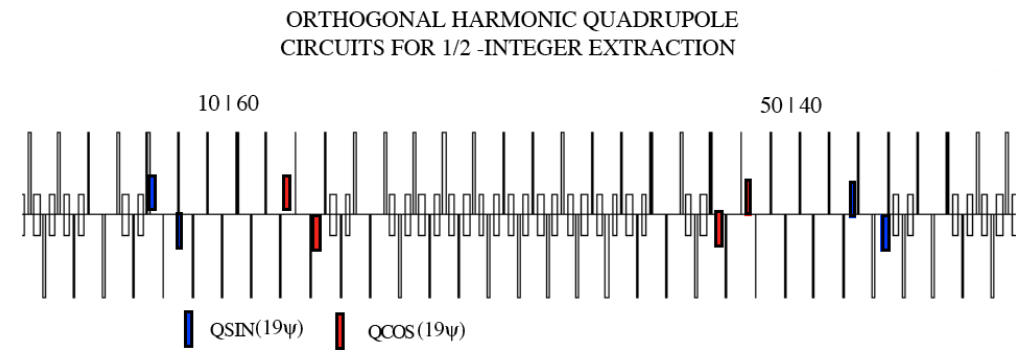

Figure 15: Location of harmonic quadrupoles on two orthogonal circuits for the half-integer configuration in which the straight sections' cells have been set individually to $60^{\circ}$, as in the arcs. 

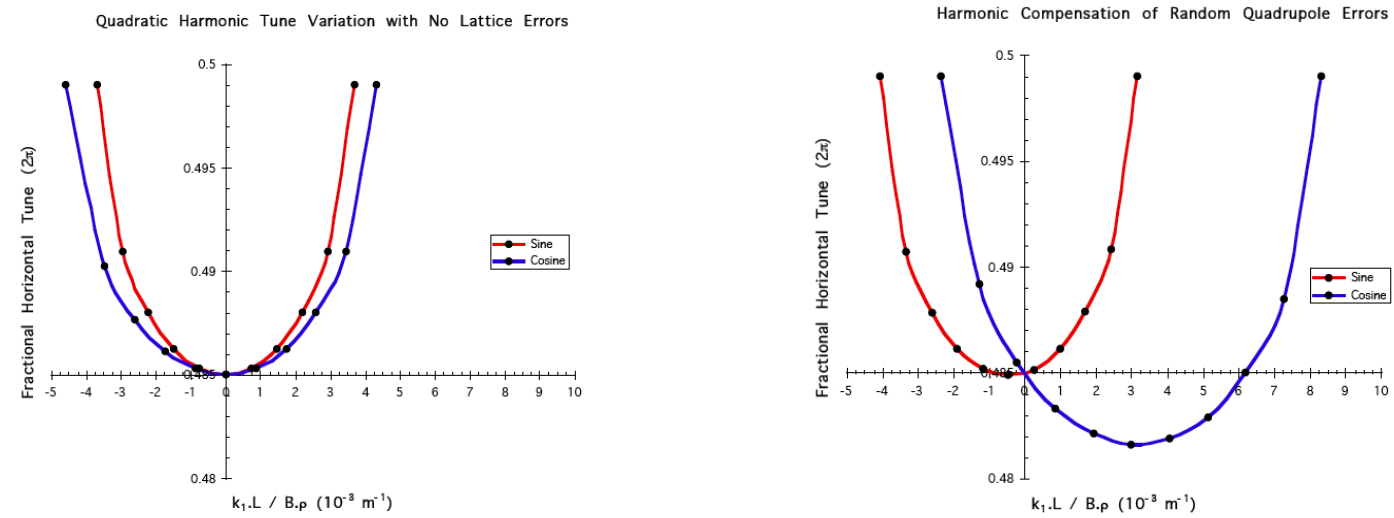

Figure 16: After introducing errors, harmonic quadrupoles circuits are tuned by observing their effect on $v_{x}$. The left and right figures show that behavior before and after random field errors are scattered into the quadrupoles.

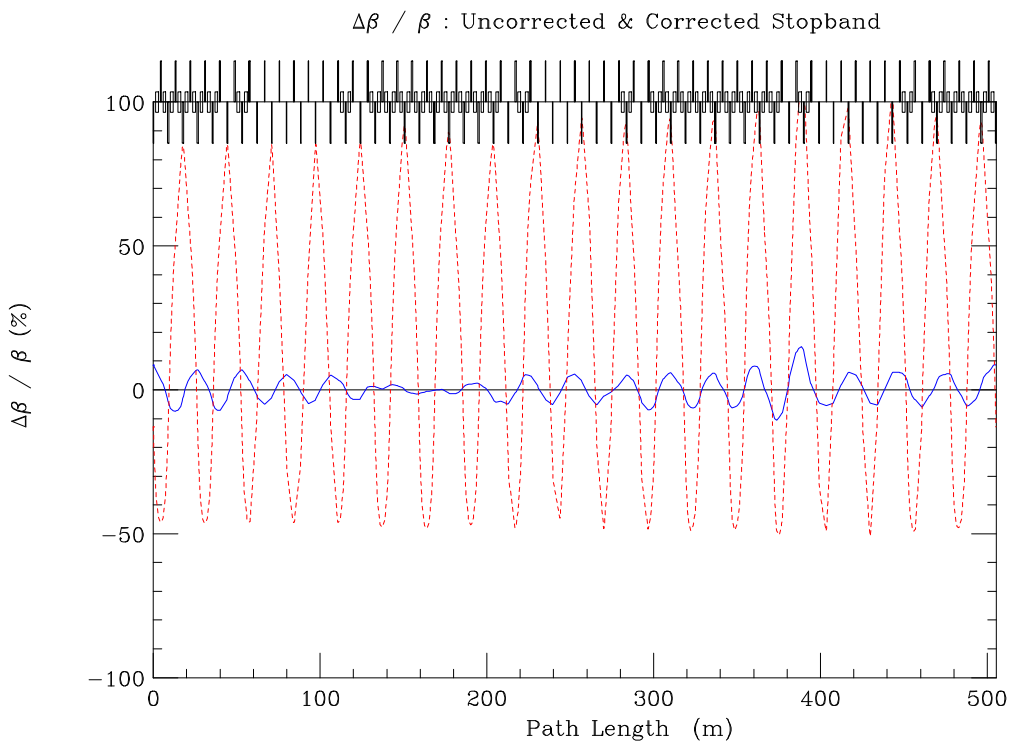

Figure 17: $\Delta \beta / \beta$ before (red) and after (blue) tuning harmonic quadrupole circuits. 


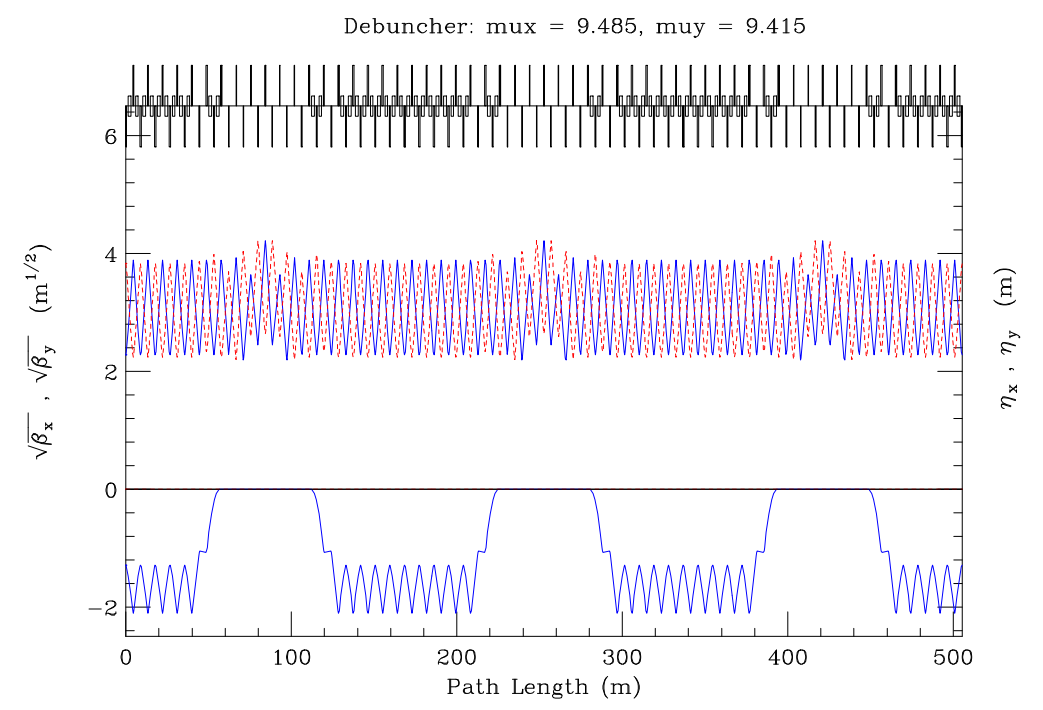

Figure 18: Lattice functions of Debuncher modified for half-integer extraction, after tuning quadrupole harmonic circuits.

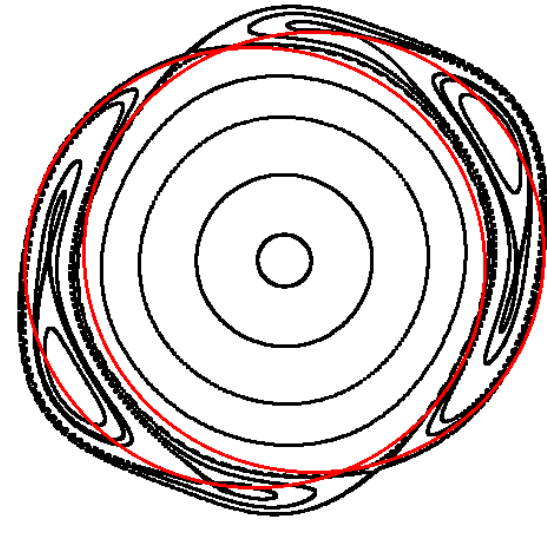

$v_{x}=9.46$

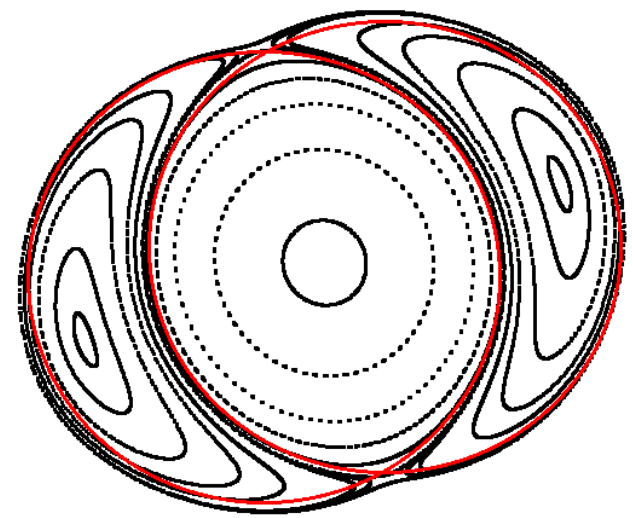

$v_{x}=9.49$

Figure 19: Two separatrices observed while using a minimalist configuration for the half-integer resonance. As expected, correspondence is better closer to the resonance, though correcting the orientation requires tuning the harmonic quadrupoles. 


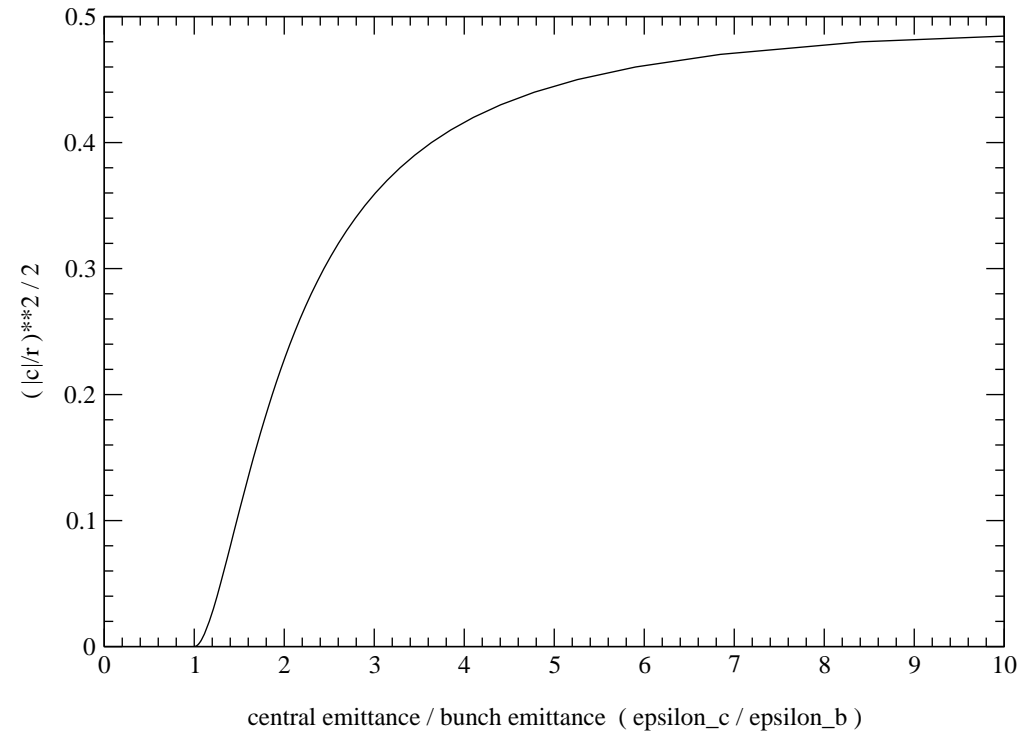

Figure 20: This had to do with quadrupole settings needed to control the shape of the half-integer separatrix. Plotted is $\left|G_{2} / \Delta\right|$ versus $\varepsilon_{c} / \varepsilon_{b}$, the reciprocal of the ratio appearing on the left hand side of Eq.(24). I don't want to talk about it. 

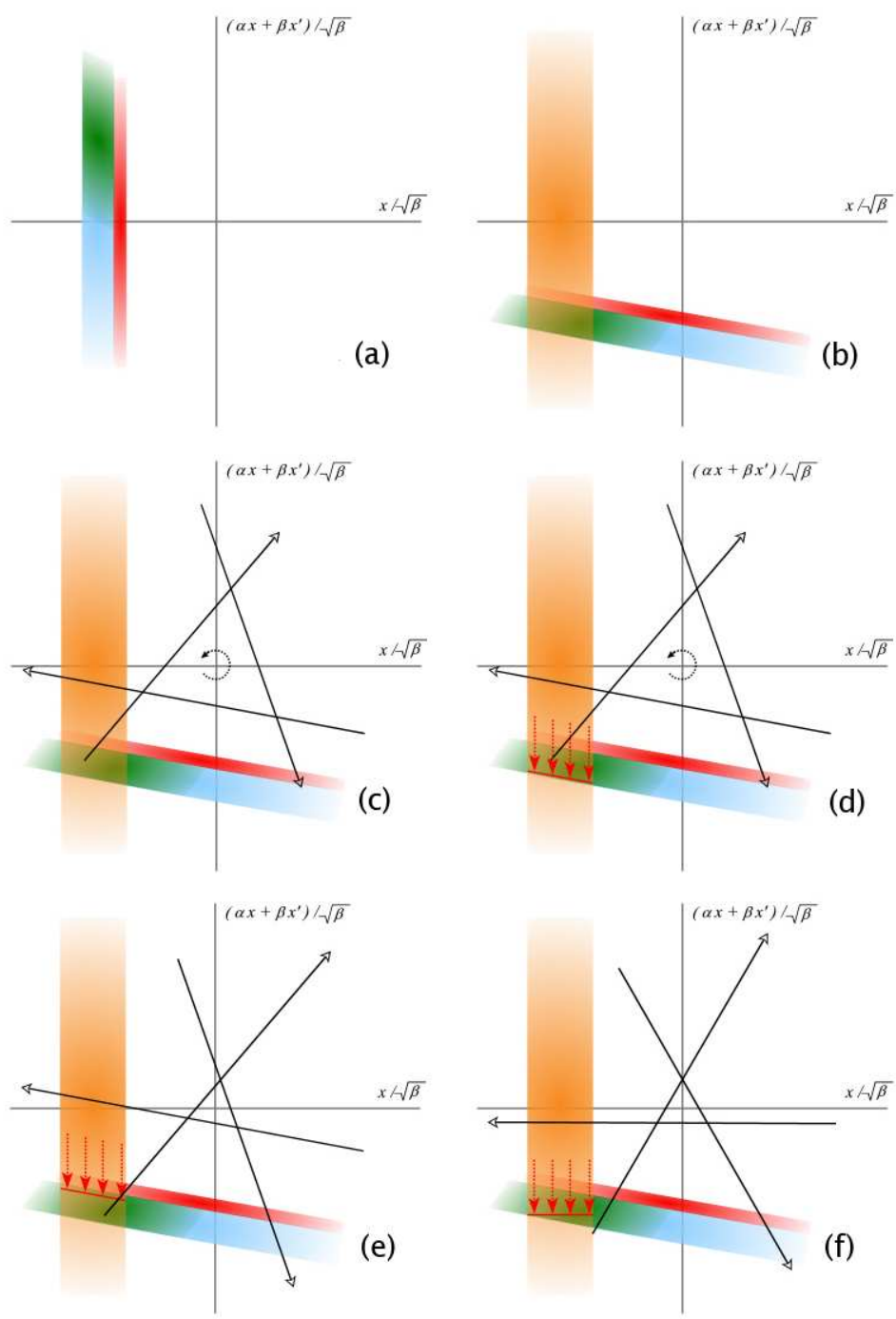

Figure 21: Conceptual diagram of third-integer extraction. 

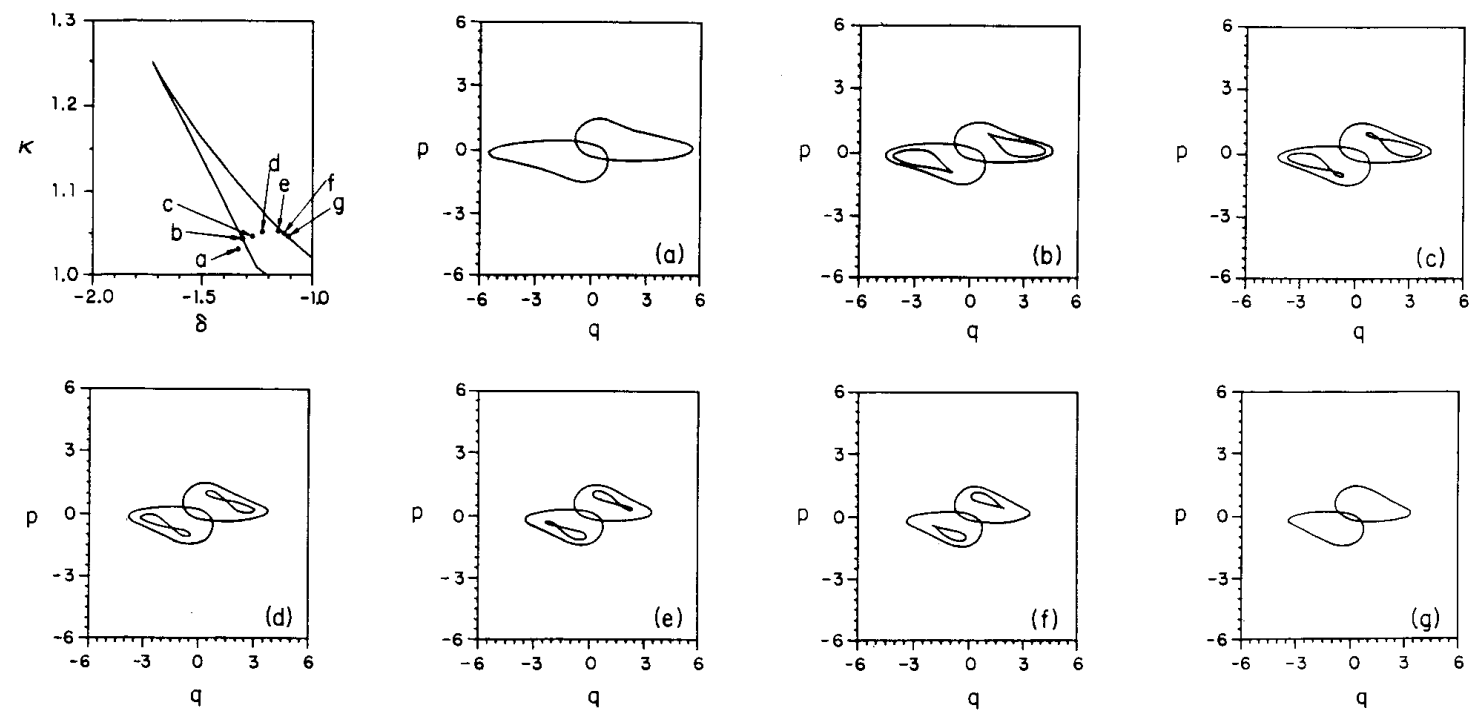

Figure 22: One class of possible separatrices arising from principal resonances excited quadrupoles and octupoles. The figure in the upper left identifies points in the space of excitation parameters that produce the separatrices seen in the other frames. (Reproduced from References [8] and [5] with permission of the author.)

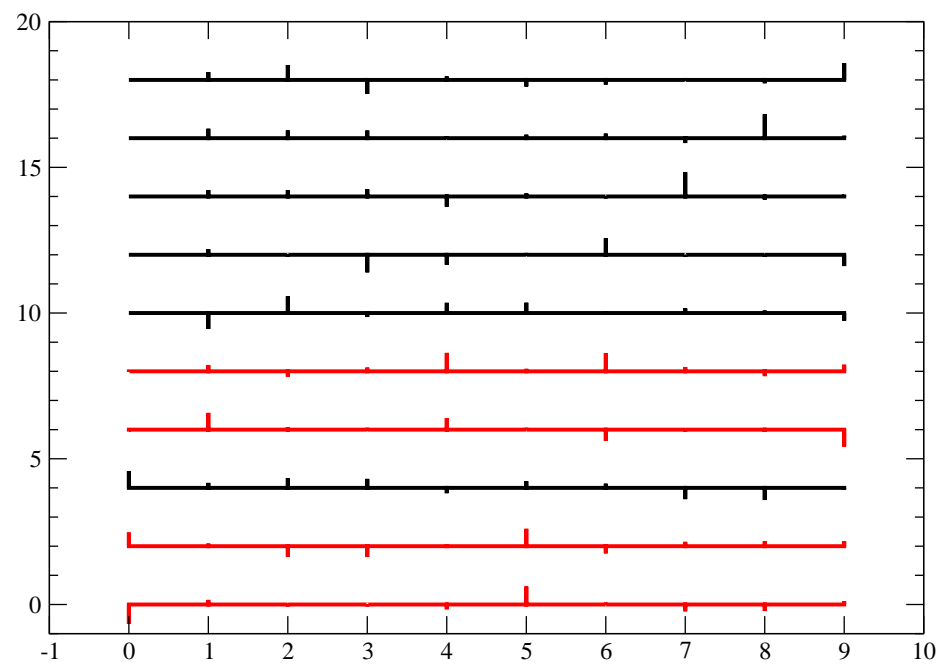

LPJM

Figure 23: Relative strengths of octupoles in circuits set according to SVD analysis. The four colored red would set up orthogonal circuits for second order and fourth order harmonics - i.e. $N / 2$ and N/4 - and must not be used in order to avoid exciting those terms. Any linear combination of the rest (colored black) would set up a zeroth harmonic octupole circuit. 